\title{
Under Different Priors \&Two Loss Functions To Compare Bayes Estimators With Some of Classical Estimators For the Parameter of Exponential Distribution
}

Dr. Jinan Abbas Naser Al-obedy -Assistant professor

Technical College of Management-Baghdad

\author{
تاريخ التقليم:2016/9/27 \\ تاريخ القبول:2016/11/28
}

\section{Abstract}

In this study, different estimators were used for estimating parameter of the exponential distribution, such as maximum likelihood estimator, moment estimator and the Bayes estimator, by assuming six types when the prior distribution for the scale parameter is: Levy distribution, Gumbel type-II distribution, Inverse Chi-square distribution, Inverted Gamma distribution, improper distribution, Non-informative distribution .Under squared and weighted squared error loss functions. We used simulation technique, to compare the performance for each estimator, several cases from Exponential distribution for data generating, for different samples sizes (small, medium, and large). Simulation results shown that The best method is the bayes estimation according to the smallest values of MSE \& MWSE for all samples sizes (n) comparative to the estimated values by using Maximum likelihood estimation method (MLE) and Moment estimation method (ME). According to obtained results, we see that when the prior distribution for $\theta$ is Inverted Gamma distribution for some values of the parameters $\alpha \& \beta$, given the best results according to the smallest values of MSE \& MWSE comparative to the same values which obtained by using MLE\& ME for the assuming true values by $\theta=0.5$ and for all samples sizes. When the prior distribution for $\theta$ is Improper distribution for some values of the parameters a $\& b$, given the best results according to the smallest values of MSE \& MWSE comparative to the same values which obtained by using MLE \& ME for the assuming true values by $\theta=1,2,4$ and all samples sizes.

Key words: The Exponential, Maximum likelihood method, Moment estimation method, Bayes method, mean squared errors (MSE), mean weighted squared errors (MWSE).

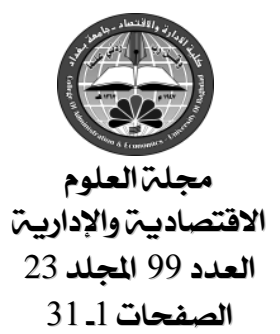




\section{Under Different Priors \& Two Loss Functions To Compare Bayes \\ Estimators With Some of Classical Estimators For the \\ Parameter of Exponential Distribution}

\section{Introduction}

The difference between Maximum Likelihood estimation and Bayesian estimation is that in maximum likelihood estimation the parameters are not random variables. In Bayesian analysis the unknown parameter is regarded as being the value of a random variable from a given probability distribution, with the knowledge of some information about its value prior to observing the data $x_{1}$, $x_{2} \ldots x_{n}$ (Ross, 2009) [9] ; we mention some of studies in a brief manner:

In (1998) Rossman, Short, and Parks [10] presented some thought provoking insights on the relationship between Bayesian and classical estimation using the continuous uniform distribution.

In (2001) Elfessi and Reineke [7] intended to explore these relationships using the exponential distribution. They show how the classical estimators can be obtained from various choices made within a Bayesian framework.

In (2005) Ali and Woo and Nadarajah [5] considered bayes estimators of the parameter of the standard exponential distribution. They derived bayes estimators under a symmetric squared error loss function as well as an asymmetric loss function.

In (2005) Al_Kutubi ${ }^{[2]}$ studied the extension of Jeffery prior information with square error loss function in exponential distribution.

In (2007) Abu-Taleb and Smadi and Alawneh [1] considered exponential survival time with the exponential random censor time. They derive bayes estimates assuming the inverted gamma prior along with the bayesian credible intervals.

In (2009) Al Kutubi and Ibrahim [3] provided the extension of Jeffery prior information with new loss function for estimating the parameter of exponential distribution of life time .Through simulation study the performance of their estimator was compared to the standard bayes with Jeffery Prior information with respect to the mean square error (MSE) and mean percentage error (MPE).

In (2009) Al_Kutubi and Ibrahim [4] annexed Jeffery prior information to get the modify bayes estimator and then compared it with standard Bayes estimator and maximum likelihood estimator to find the best (less MSE and MPE). They derived Bayesian and Maximum likelihood of the scale parameter and survival functions. Simulation study was used to compare between estimators and Mean Square Error (MSE) and Mean Percentage Error (MPE) of estimators are computed.

In (2010) Tahir and Aslam [11] provided the comparison of uninformative (Jeffrey's and uniform) priors for the parameter of the exponential model for time-to-failure data. They also presented Bayesian and classical analysis of the model. Their comparison is based upon the posterior variance, the bayesian point and interval estimates, the coefficients of skewness of the posterior distribution and the posterior predictive distribution.

In (2013) Yang and Zhou and Yuan [15] studied the bayes estimation of parameter of exponential distribution under a bounded loss function, named reflected gamma loss function, which proposed by Towhidi and Behboodian (1999). They used the inverse Gamma prior distribution as the prior distribution of the parameter of exponential distribution. Bayesian estimators are obtained under squared error loss and the reflected gamma loss functions. 


\section{Under Different Priors \& Two Loss Functions To Compare Bayes Estimators With Some of Classical Estimators For the Parameter of Exponential Distribution}

\section{The aim of research}

The objective of this research, we try to find best method to estimate parameter of exponential distribution .According to the smallest value of Mean Square Errors (MSE) and Mean Weighted Square Errors (MWSE) were calculated to compare the methods of estimation. We used the maximum likelihood estimator, the moment estimator and the bayes estimator by assuming six types of priors, to get bayes estimation: Levy distribution, Gumbel type-II distribution, Inverse Chi-square distribution, Inverted Gamma distribution, Improper distribution, and Non-informative distribution when the Bayesian estimation based on squared and weighted squared error loss functions.

Several cases from exponential distribution for data generating ,of different samples sizes (small, medium, and large).The results were obtained by using simulation technique, Programs written using MATLAB-R2008a program were used.

\subsection{Exponential Distribution}

Let us consider $x_{1}, x_{2}, \ldots, x_{n}$ is a random sample of $n$ independent observations from an exponential distribution having the probability density function (pdf) define as [7,8]:

$$
\mathrm{f}(\mathrm{x} ; \theta)=\theta^{-1} \exp \left(-\frac{\mathrm{x}}{\theta}\right) \quad, \quad \mathrm{x}>0
$$

where $\theta>0$ is mean, standard deviation, and scale parameter of the distribution, $\theta$ is a survival parameter in the sense that if a random variable $\mathbf{x}$ is the duration of time that a given biological or mechanical system manages to survive and $\mathbf{x} \sim \operatorname{Exp}(\theta)$ then $\mathbf{E}[\mathbf{x}]=\theta$. That is to say, the expected duration of survival of the system is $\theta$ units of time.

\subsection{Parameter Estimation Methods}

In this section, we used several methods to estimation parameter $\theta$.

\subsubsection{Maximum likelihood Estimation(MLE)}

From the Exponential pdf given in (1) the likelihood function will be as follows[6]:

$$
\mathrm{L}(\underline{\mathrm{x}} \backslash \theta)=\prod_{\mathrm{i}=1}^{\mathrm{n}} \mathrm{f}(\mathrm{x} ; \theta)=\theta^{-\mathrm{n}} \exp \left(-\frac{\sum_{\mathrm{i}=1}^{\mathrm{n}} \mathrm{x}_{\mathrm{i}}}{\theta}\right)
$$

By taking the log and differentiating partially with respect to $\theta$, we get:

$$
\frac{\partial}{\partial \theta} \log \mathrm{L}(\underline{\mathrm{x}} \backslash \theta)=-\frac{\mathrm{n}}{\theta}+\frac{\sum_{\mathrm{i}=1}^{\mathrm{n}} \mathrm{x}_{\mathrm{i}}}{\theta^{2}}
$$

Then the MLE of $\theta$ is the solution of equation (2) after equating the first derivative to zero, Hence:

$\hat{\theta}_{\mathrm{MLE}}=\frac{\sum_{\mathrm{i}=1}^{\mathrm{n}} \mathrm{x}_{\mathrm{i}}}{\mathrm{n}}=\overline{\mathrm{x}}$ 


\section{Under Different Priors \&Two Loss Functions To Compare Bayes}

\subsubsection{Moments Estimation (ME)}

The method of moments is another technique commonly used in the field of estimation of parameters. If $\underline{x}=\left(x_{1}, x_{2}, \ldots, x_{n}\right)$ be a random sample of size (n) represent a set of data, then an unbiased estimator for the $r^{\text {th }}$ origin moment is [6]:

$\mathrm{m}_{\mathrm{r}}=\frac{\sum_{\mathrm{i}=1}^{\mathrm{n}} \mathrm{x}_{\mathrm{i}}^{\mathrm{r}}}{\mathrm{n}}$

Where $m_{r}$ stands for the $r^{\text {th }}$ sample moment. The first moment of the Exponential distribution as:

$$
\mathrm{M}_{1}=\mathrm{E}(\mathrm{x})=\frac{1}{(1 / \theta)}=\theta
$$

Therefore by equating sample and population moments we get

$$
\mathrm{m}_{1}=\mathrm{M}_{1}=\mathrm{E}(\mathrm{x})=\frac{1}{(1 / \theta)}=\theta
$$

$$
\text { From (7) we get } \bar{x}=\theta \Rightarrow \hat{\theta}_{M M}=\bar{x}
$$

\subsubsection{Bayes Estimation Method}

Let $\underline{x}=\left(x_{1}, x_{2}, \ldots, x_{n}\right)$ be a random sample of size $n$ with probability density function given in equation (1) and likelihood function given in equation (2).In this paper, we derived the posterior distributions for the unknown parameter $\theta$ using the following six types of priors, and then get bayes estimation [8]:

1. Levy distribution.

2. Gumbel type-II distribution [12].

3. Inverse Chi-square distribution [14].

4. Inverted Gamma distribution [13].

5. Improper distribution.

6. Non-informative distribution.

2.3.3.1 The posterior distribution using different Priors

In this section, we derive the posterior distributions.It is assumed that $\theta$ follows six types of prior distributions with pdf as given in table below: 
Table -1: The six types of prior distributions $(P(\theta))$ with pdf for $\theta$.

\begin{tabular}{|c|c|}
\hline Prior distribution & $\mathrm{P}(\theta)$ \\
\hline$\theta \sim \operatorname{Levy}\left(b_{3}\right)$ & $P(\theta) \alpha \sqrt{\frac{b_{3}}{2 \pi}} \theta^{-\frac{3}{2}} \exp \left(-\frac{b_{3}}{2 \theta}\right)$ for $b_{3}, \theta>0$ \\
\hline$\theta \sim$ Gumbel type-II( b ) & $P(\theta) \alpha \quad b \theta^{-2} \exp \left(-\frac{b}{\theta}\right)$ for $\quad b, \theta>0$ \\
\hline$\theta \sim$ Inverse Chi-square( v ) & $\mathrm{P}(\theta) \alpha \frac{1}{2^{\frac{\mathrm{v}}{2}}} \theta^{-\frac{\mathrm{v}}{2}-1} \exp \left(-\frac{1}{2 \theta}\right)$ for $\mathrm{v}, \theta>0$ \\
\hline$\theta \sim \operatorname{Inverted} \operatorname{Gamma}(\alpha, \beta)$ & $\mathrm{P}(\theta) \alpha \frac{\beta^{\alpha}}{\Gamma \alpha} \theta^{-(\alpha+1)} \exp \left(-\frac{\beta}{\theta}\right)$ for $\alpha, \beta, \theta>0$ \\
\hline$\theta \sim \operatorname{Improper}(\mathrm{a}, \mathrm{b})$ & $\begin{array}{l}\mathrm{P}(\theta) \alpha \theta^{-(\mathrm{a}+1)} \exp \left(-\frac{\mathrm{b}}{\theta}\right) \text { for } \mathrm{b}, \theta>0 \\
\quad \text { and }-\infty<\mathrm{a}<\infty\end{array}$ \\
\hline$\theta \sim$ Non-informative( $\mathrm{c}$ ) & $\mathrm{P}(\theta) \alpha \frac{1}{\theta^{\mathrm{c}}}$ for $\theta, \mathrm{c}>0$ \\
\hline
\end{tabular}

Then the posterior distribution of given the data $\underline{x}=\left(x_{1}, x_{2}, \ldots, x_{n}\right)$ is[7]:

$$
\mathrm{P}(\theta \backslash \mathrm{x})=\frac{\mathrm{L}(\underline{\mathrm{x}} \backslash \theta) \mathrm{P}(\theta)}{\int_{\theta} \mathrm{L}(\underline{\mathrm{x}} \backslash \theta) \mathrm{P}(\theta) \mathrm{d} \theta}
$$

Substituting the equation (2) and for each $P(\theta)$ as shown in table -1 in equation (9), we get the posterior distributions for the unknown parameter $\theta$ are derived using the following six types of priors ( for more details see Appendix-A). 


\section{Under Different Priors \&Two Loss Functions To Compare Bayes Estimators With Some of Classical Estimators For the Parameter of Exponential Distribution}

Table -2: The posterior distributions $(P(\theta \backslash x))$ for the unknown parameter $(\theta)$ are derived using the following six types of priors.

\begin{tabular}{|c|c|}
\hline Prior dist ${ }^{\mathrm{n}}$. & The posterior distribution $(P(\theta \backslash x))$ \\
\hline Levy & 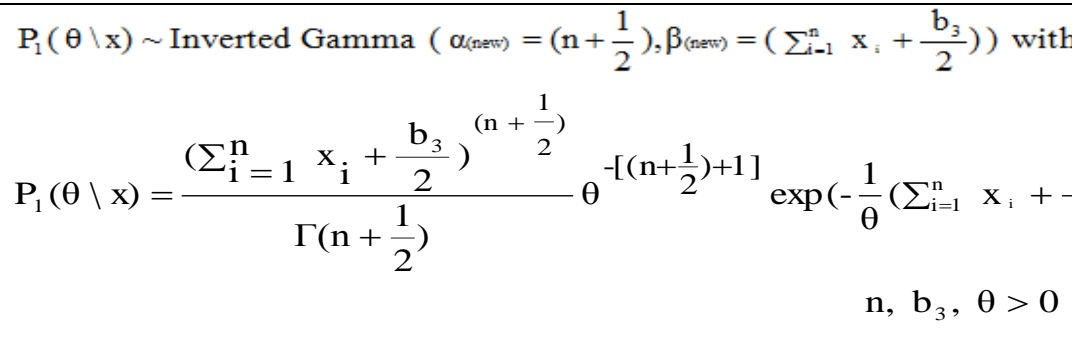 \\
\hline \multirow[t]{2}{*}{ Gumbel type-II } & \multirow{2}{*}{$\begin{array}{r}\mathrm{P}_{2}(\theta \backslash \mathrm{x}) \sim \text { Inverted Gamma }\left(\alpha_{\text {(new) }}=(\mathrm{n}+1), \beta_{\text {(new) }}=\left(\sum_{\mathrm{i}-1}^{\mathrm{n}} \mathrm{x}_{\mathrm{i}}+\mathrm{b}\right)\right) \text { with } \\
\mathrm{P}_{2}(\theta \backslash \mathrm{x})=\frac{\left(\sum_{\mathrm{i}=1}^{\mathrm{n}} \mathrm{x}_{\mathrm{i}}+\mathrm{b}\right)^{(\mathrm{n}+1)} \theta^{-[(\mathrm{n}+1)+1]} \exp \left(-\frac{1}{\theta}\left(\sum_{\mathrm{i}=1}^{\mathrm{n}} \mathrm{x}_{\mathrm{i}}+\mathrm{b}\right)\right)}{\Gamma(\mathrm{n}+1)} \\
\mathrm{n}, \mathrm{b}, \theta>0\end{array}$} \\
\hline & \\
\hline Inverse Chi-square & 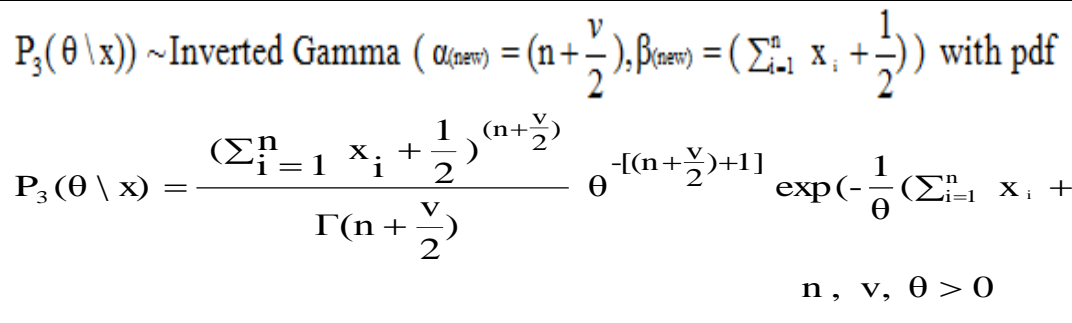 \\
\hline \multirow[t]{2}{*}{ Inverted Gamma } & \multirow{2}{*}{ 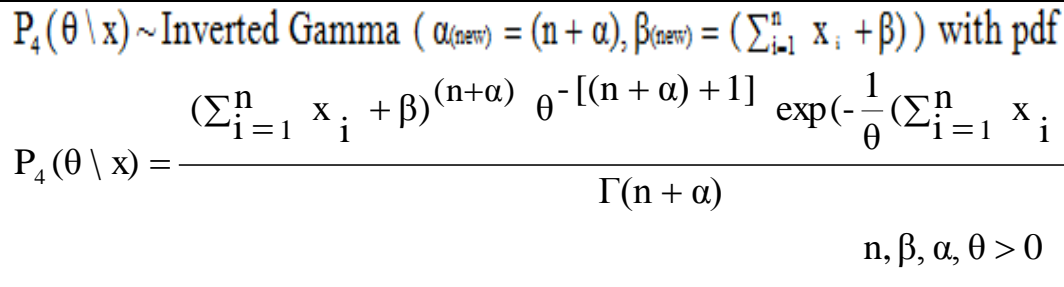 } \\
\hline & \\
\hline Improper & $\begin{array}{c}\mathrm{P}_{5}(\theta \backslash \mathrm{x}) \sim \text { Inverted Gamma }\left(\alpha_{(\mathrm{naxi})}=(\mathrm{n}+\mathrm{a}), \beta_{\text {(nari) }}=\left(\sum_{\mathrm{i}=1}^{\mathrm{n}} \mathrm{x}_{\mathrm{i}}+\mathrm{b}\right)\right) \text { with pdf } \\
\mathrm{P}_{5}(\theta \backslash \mathrm{x})=\frac{\left(\sum_{\mathrm{i}=1}^{\mathrm{n}} \mathrm{x}_{\mathrm{i}}+\mathrm{b}\right)^{(\mathrm{n}+\mathrm{a})} \theta^{-[(\mathrm{n}+\mathrm{a})+1]} \exp \left(-\frac{1}{\theta}\left(\sum_{\mathrm{i}=1}^{\mathrm{n}} \mathrm{x}_{\mathrm{i}}+\mathrm{b}\right)\right)}{\Gamma(\mathrm{n}+\mathrm{a})} \\
\mathrm{n}, \mathrm{b}, \theta>0 \text { and }-\infty<\mathrm{a}<\infty\end{array}$ \\
\hline
\end{tabular}




\section{Under Different Priors \&Two Loss Functions To Compare Bayes Estimators With Some of Classical Estimators For the Parameter of Exponential Distribution}

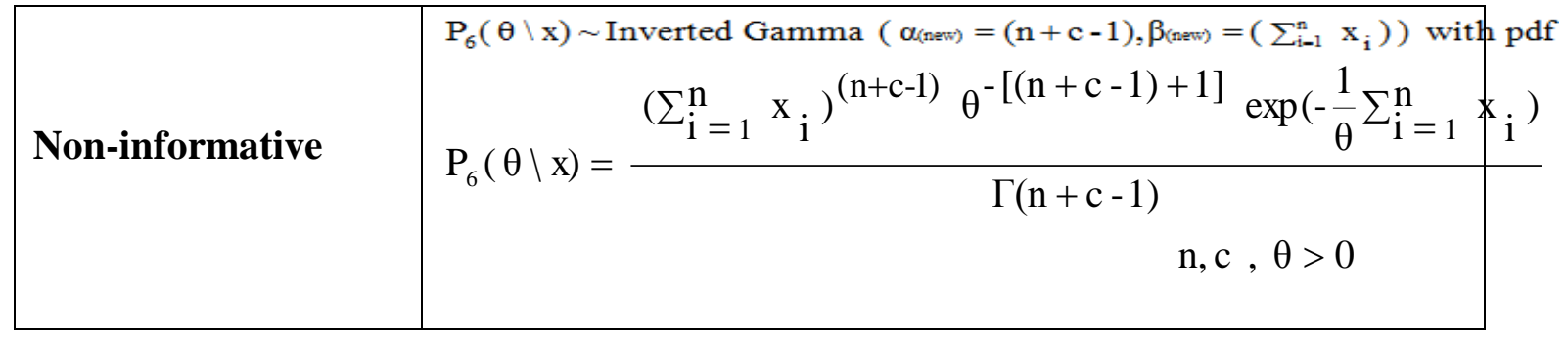

\subsubsection{Bayes' Estimators}

In this section, we derive Bayes' estimators for the scale parameter $\theta$, it was considered with six different priors and under two loss functions:

1. The squared error loss function $L_{1}(\hat{\theta}, \theta)=(\hat{\theta}-\theta)^{2}$.

2. The weighted squared error loss function $L_{2}(\hat{\theta}, \theta)=\frac{(\hat{\theta}-\theta)^{2}}{\theta}$.

Where $\theta$ is an estimator for $\theta$, was considered with different six priors, and under two loss functions. Following is the derivation of these estimators:

\section{First: The squared error loss function}

In this section, we derive Bayes' estimator. To obtain the Bayes' estimator, we minimize the posterior expected loss given by:

$$
\mathrm{L}_{1}(\hat{\theta}, \theta)=(\hat{\theta}-\theta)^{2}
$$

After simplified steps, we get Bayes estimator of $\theta$ denoted by $\hat{\theta}_{\mathrm{SE}}$ for the above prior as follows

$\hat{\theta}_{\mathrm{SE}}=\mathrm{E}(\theta \backslash \mathrm{x})=\int_{0}^{\infty} \theta \mathrm{P}(\theta \backslash \mathrm{x}) \mathrm{d} \theta$

So, the following results are the derivations of these estimators under the squared error loss function with different six priors (for more details see Appendix-B). 
Table -3: The estimators ( $\left.\hat{\theta}_{\mathrm{SE}}\right)$ under the squared error loss function with different six priors.

\begin{tabular}{|l|l|}
\hline Prior distribution & $\hat{\theta}_{\mathrm{SE}}=\mathrm{E}(\theta \backslash \mathrm{x})=\int_{0}^{\infty} \theta \mathrm{P}(\theta \backslash \mathrm{x}) \mathrm{d} \theta$ \\
\hline Levy & $\hat{\theta}_{\mathrm{SE} 1}=\frac{\Gamma\left(\mathrm{n}-\frac{1}{2}\right)}{\Gamma\left(\mathrm{n}+\frac{1}{2}\right)}\left(\sum_{\mathrm{i}=1}^{\mathrm{n}} \mathrm{x}_{\mathrm{i}}+\frac{\mathrm{b}_{3}}{2}\right), \mathrm{n} \& \mathrm{~b}_{3}>0$ \\
\hline Gumbel type-II & $\hat{\theta}_{\mathrm{SE} 2}=\frac{\Gamma(\mathrm{n})}{\Gamma(\mathrm{n}+1)}\left(\sum_{\mathrm{i}=1}^{\mathrm{n}} \mathrm{x}_{\mathrm{i}}+\mathrm{b}\right), \mathrm{n} \& \mathrm{~b}>0$ \\
\hline Inverse Chi- & $\hat{\theta}_{\mathrm{SE} 3}=\frac{\Gamma\left(\mathrm{n}+\frac{\mathrm{v}}{2}-1\right)}{\Gamma\left(\mathrm{n}+\frac{\mathrm{v}}{2}\right)}\left(\sum_{\mathrm{i}=1}^{\mathrm{n}} \mathrm{x}_{\mathrm{i}}+\frac{1}{2}\right), \mathrm{n} \& \mathrm{v}>0$ \\
\hline Inverted Gamma & $\hat{\theta}_{\mathrm{SE} 4}=\frac{\Gamma(\mathrm{n}+\alpha-1)}{\Gamma(\mathrm{n}+\alpha)}\left(\sum_{\mathrm{i}=1}^{\mathrm{n}} \mathrm{x}_{\mathrm{i}}+\beta\right), \mathrm{n}, \beta, \alpha>0$ \\
\hline Improper & $\hat{\theta}_{\mathrm{SE} 5}=\frac{\Gamma(\mathrm{n}+\mathrm{a}-1)}{\Gamma(\mathrm{n}+\mathrm{a})}\left(\sum_{\mathrm{i}=1}^{\mathrm{n}} \mathrm{x}_{\mathrm{i}}+\mathrm{b}\right), \mathrm{n}, \mathrm{b}, \mathrm{a}>0$ \\
\hline Non-informative & $\hat{\theta}_{\mathrm{SE} 6}=\frac{\Gamma(\mathrm{n}+\mathrm{c}-2)}{\Gamma(\mathrm{n}+\mathrm{c}-1)}\left(\sum_{\mathrm{i}=1}^{\mathrm{n}} \mathrm{x}_{\mathrm{i}}\right), \mathrm{n}, \mathrm{c}>0$ \\
\hline
\end{tabular}

\section{Second: The weighted squared error loss function}

In this section, we derive Bayes' estimator .To obtain the Bayes' estimator, we minimize the posterior expected loss given by:

$\mathrm{L}_{2}(\hat{\theta}, \theta)=\frac{(\hat{\theta}-\theta)^{2}}{\theta}$

After simplified steps, we get Bayes estimator of $\theta$ denoted by $\hat{\theta}_{\mathrm{WSE}}$ for the above prior as follows

$\hat{\theta}_{\mathrm{wSE}}=\frac{1}{\mathrm{E}\left(\frac{1}{\theta} \backslash \mathrm{x}\right)}=\frac{1}{\int_{0}^{\infty} \frac{1}{\theta} \mathrm{P}(\theta \backslash \mathrm{x}) \mathrm{d} \theta}$

So, the following results are the derivations of these estimators under the weighted squared error loss function with different six priors (for more details see Appendix-C). 


\section{Under Different Priors \&Two Loss Functions To Compare Bayes}

Estimators With Some of Classical Estimators For the Parameter of Exponential Distribution

Table -4: The estimators ( $\theta_{\mathrm{WSE}}$ ) under the weighted squared error loss function with different six priors.

\begin{tabular}{|c|c|c|}
\hline Prior distribution & $\hat{\theta}_{\mathrm{wSE}}=\frac{1}{\mathrm{E}\left(\frac{1}{\theta} \backslash \mathrm{x}\right)}=\frac{1}{\int_{0}^{\infty} \frac{1}{\theta} \mathrm{P}(\theta \backslash \mathrm{x}) \mathrm{d} \theta}$ & \\
\hline Levy & $\hat{\theta}_{\mathrm{wSEl}}=\frac{\Gamma\left(\mathrm{n}+\frac{1}{2}\right)\left(\sum_{\mathrm{i}=1}^{\mathrm{n}} \mathrm{x}_{\mathrm{i}}+\frac{\mathrm{b}_{3}}{2}\right)}{\Gamma\left(\mathrm{n}+\frac{3}{2}\right)}$ &, $\mathrm{n} \& \mathrm{~b}_{3}>0$ \\
\hline Gumbel type-II & $\hat{\theta}_{\mathrm{WSE} 2}=\frac{\Gamma(\mathrm{n}+1)\left(\sum_{\mathrm{i}=1}^{\mathrm{n}} \mathrm{x}_{\mathrm{i}}+\mathrm{b}\right)}{\Gamma(\mathrm{n}+2)}$ & , $\mathrm{n} \& \mathrm{~b}>0$ \\
\hline $\begin{array}{l}\text { Inverse Chi- } \\
\text { square }\end{array}$ & $\hat{\theta}_{\mathrm{WSE} 3}=\frac{\Gamma\left(\mathrm{n}+\frac{\mathrm{v}}{2}\right)\left(\sum_{\mathrm{i}=1}^{\mathrm{n}} \mathrm{x}_{\mathrm{i}}+\frac{1}{2}\right)}{\Gamma\left(\mathrm{n}+\frac{\mathrm{v}}{2}+1\right)}$ & $\mathrm{n}, \mathrm{v}>0$ \\
\hline Inverted Gamma & $\hat{\theta}_{\mathrm{wSE} 4}=\frac{\Gamma(\mathrm{n}+\alpha)\left(\sum_{\mathrm{i}=1}^{\mathrm{n}} \mathrm{x}_{\mathrm{i}}+\beta\right)}{\Gamma(\mathrm{n}+\alpha+1)}$ & $\mathrm{n}, \beta, \alpha>0$ \\
\hline Improper & $\hat{\theta}_{\mathrm{WSES}}=\frac{\Gamma(\mathrm{n}+\mathrm{a})\left(\sum_{\mathrm{i}=1}^{\mathrm{n}} \mathrm{x}_{\mathrm{i}}+\mathrm{b}\right)}{\Gamma(\mathrm{n}+\mathrm{a}+1)}$ & $\mathrm{n}, \mathrm{b}, \mathrm{a}>0$ \\
\hline Non-informative & $\hat{\theta}_{\mathrm{WSE} 6}=\frac{\Gamma(\mathrm{n}+\mathrm{c}-1)\left(\sum_{\mathrm{i}=1}^{\mathrm{n}} \mathrm{x}_{\mathrm{i}}\right)}{\Gamma(\mathrm{n}+\mathrm{c})}$ & $\mathrm{n}, \mathrm{c}>0$ \\
\hline
\end{tabular}

\section{Simulation Study}

In this study, we have generated random samples from Exponential distribution and compared the performance of MLE and MME and Bayes estimator based on them. So we have considered several steps to perform simulation study as follow:

1. We have chosen sample size $n=10,25,50$ and 100 to represent small, moderate and large sample size.

2. We generated data from exponential distribution for the scale parameter; we have considered randomly several values for the parameter of exponential distribution $\theta=0.5,1,2,4$.

3. We used randomly three values for the parameter of the Levy distribution $\left(b_{3}=0.5,1,2\right)$ as prior distribution for $\theta$.

4. We used randomly three values for the parameter of the Gumbel type-II distribution $(b=2,3,5)$ as prior distribution for $\theta$. 


\section{Under Different Priors \& Two Loss Functions To Compare Bayes Estimators With Some of Classical Estimators For the Parameter of Exponential Distribution}

5. We used randomly three values for the parameter of the Inverse Chi-square distribution $(v=2,4,6)$ as prior distribution for $\theta$.

6. We used randomly two values for the parameters of the Inverted Gamma distribution $(\alpha=2,3 \& \beta=0.5,1)$ as prior distribution for $\theta$.

7. We used randomly three values for the parameters of the Improper distribution ( $a=1,2,3 \& b=1,2,3)$ as prior distribution for $\theta$.

8. We used randomly three values for the function of the non-informative prior distribution $\mathrm{c}=1,2,3$.

9. The number of replication used was $(r=1000)$ for each sample size $(n)$.

10. We obtained estimators for scale parameter from equations (4), (8) and also

the estimators in table $\mathbf{- 3}$, it means the estimators $\left(\theta_{\mathrm{SE}}\right)$ under the squared error loss function with six different priors .And the estimators in table $\mathbf{- 4}$, it means the estimators $\left(\hat{\theta}_{\mathrm{WSE}}\right)$ under the weighted squared error loss function with different six priors.

The simulation program was written by using MATLAB-R2008a program. After the parameter $\theta$ was estimated, Mean Square Errors (MSE) and Mean weighted squared Errors (MWSE) were calculated to compare the methods of estimation, where:

- $\mathrm{MSE}=\frac{1}{\mathrm{r}} \sum_{\mathrm{r}=1}^{1000}(\hat{\theta}(\mathrm{r})-\theta)^{2}$

- $\mathrm{MWSE}=\frac{1}{\mathrm{r}} \sum_{\mathrm{r}=1}^{1000}\left[(\hat{\theta}(\mathrm{r})-\theta)^{2} / \theta\right]$

See appendix-D, for the programs algorithm. The results of the simulation study are summarized and tabulated in tables (4.1-4.4).In each row of tables (4.14.4), we have four estimated values for $\theta(\theta)$ with MSE for all samples sizes (n) and values ( $\left.b_{3}, b, v, \alpha, \beta, a, b, c\right)$ respectively. Also the results of the simulation study are summarized and tabulated in tables (4.5-4.8).In each row of tables (4.5-4.8), we have four estimated values for $\theta(\theta)$ with MWSE for all samples sizes (n) and values $\left(b_{3}, b, v, \alpha, \beta, a, b, c,\right)$ respectively. By using different estimation methods that is maximum likelihood estimator and the moment estimator .And the Bayes estimators in six types of prior distribution .So our criteria is the best method that gives the smallest value of ( MSE ) and ( MWSE ). We list the results in the following tables (4.1 -4.8). 


\section{Under Different Priors \& Two Loss Functions To Compare Bayes Estimators With Some of Classical Estimators For the Parameter of Exponential Distribution}

In general, as we see in the tables (4.1-4.8) by using different estimation methods, we find the Mean Square Errors (MSE) and Mean weighted squared Errors (MWSE) were decreased when sample size increased in all cases .And we obtained the same results for MSE\& MWSE by using maximum likelihood estimation (MLE) and the moment estimation(ME) for all sample sizes (n), because they have the same formula see formula from equations (4), (8).

we see in the tables (4.1-4.4) that we obtained un appropriate estimated values for $\theta(\theta)$, when the prior distribution for $\theta$ is levy distribution, for all assuming values for $\mathbf{b}_{3} \&$ for the true values for $\theta=0.5,1,2,4$, and for all samples sizes (n) comparative to the estimated values by using MLE and ME, according to the smallest values of MSE for all samples sizes (n).

So we obtained over estimated values for $\theta(\theta)$, when the prior distribution for $\theta$ is Gumbel type-II distribution, for all assuming values for $b$ and $\theta=0.5,1,2,4$, and for all samples sizes (n) comparative to the estimated values by using MLE and ME, according to the smallest values of MSE for all samples sizes (n).

In table (4.1), when the true value of $\theta(\theta=0.5)$ in general, we obtained a good estimation according to the smallest values of MSE for all samples sizes (n) comparative to the estimated values by using MLE and ME .we listed them when the prior distribution for $\theta$ are

- Inverse Chi-square distribution with $v=6$.

- Inverted Gamma distribution with $(\alpha=3, \beta=1)$.

- Improper distribution with $(a=3, b=1)$.

- Non-informative distribution with $\mathrm{c}=3$.

In table (4.2), when the true value of $\theta(\theta=1)$ in general, we obtained a good estimation according to the smallest values of MSE for all samples sizes (n) ,comparative to the estimated values by using MLE and ME .we listed them when the prior distribution for $\theta$ are

- Inverse Chi-square distribution with $v=4 \& 6$.

- Inverted Gamma distribution with $(\alpha=3, \beta=1)$.

- Improper distribution with $(a=3, b=2)$.

- Non-informative distribution with $\mathrm{c}=3$.

In tables (4.3) \& (4.4), when the true value of $\theta(\theta=2) \&(\theta=4)$ in general, we obtained a good estimation according to the smallest values of MSE for all samples sizes (n), comparative to the estimated values by using MLE and ME .we listed them when the prior distribution for $\theta$ are

- Inverse Chi-square distribution with $v=4$.

- Inverted Gamma distribution with $(\alpha=2, \beta=1)$.

- Improper distribution with $(a=b=3)$.

- Non-informative distribution with $\mathrm{c}=3$.See tables (4.1-4.4) . 


\section{Under Different Priors \& Two Loss Functions To Compare Bayes Estimators With Some of Classical Estimators For the Parameter of Exponential Distribution}

Table 4.1: Shows the values for $\theta$ under Table 4.2: Shows the values for

$\theta$ under square error loss function (MSE). $\quad$ square error loss function (MSE).

\begin{tabular}{|c|c|c|c|c|c|c|c|c|c|c|c|}
\hline \multirow{3}{*}{ Method } & \multirow{2}{*}{\multicolumn{3}{|c|}{ parameters }} & \multicolumn{4}{|c|}{ Estimate for $\theta(\theta)$} & \multicolumn{4}{|c|}{ MSSE } \\
\hline & & & & \multicolumn{4}{|c|}{ Sample Size(n) } & \multicolumn{4}{|c|}{ Sample Size(n) } \\
\hline & $\theta$ & . & . & 10 & 25 & 50 & 100 & 10 & 25 & 30 & 100 \\
\hline МLE & 0.5 & . & . & 0.4918 & 0.5030 & 0.5017 & 0,5001 & 0.0225 & 0.0103 & 0.0052 & 0,0026 \\
\hline$\sqrt{\mathbb{E}}$ & 0.5 & . & W & 0.4918 & 0,5030 & 0.5017 & 0,5001 & 0.0225 & 0103 & 0,0052 & 0026 \\
\hline \multirow[t]{4}{*}{ Baves } & $\theta$ & $b_{3}$ & . & \multicolumn{8}{|c|}{ Lerv distribution $(P,(\theta \mid x))$} \\
\hline & 0.5 & 0.5 & . & 5.1683 & 12.826 & 25,333 & 50.258 & 24,035 & 158,35 & 629.56 & 2502.2 \\
\hline & & 1 & . & 5,4183 & 13,076 & 25,583 & 50.508 & 26.431 & 164.57 & 642.04 & 2527.1 \\
\hline & & 2 & . & 5.9183 & 13,576 & 26.083 & 51.008 & 31.6 & 177.4 & 667,37 & 2577.4 \\
\hline \multirow[t]{4}{*}{ Bayes } & $\theta$ & $\mathrm{b}$ & 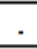 & \multicolumn{8}{|c|}{ Gumbeltrpe-Idistribution $\left(P_{1}(\theta) \times\right)$} \\
\hline & 0.5 & 2 & & 0.6918 & 0,5830 & 0.5417 & 0,5201 & 0.0592 & 0,0177 & 0.0069 & 0,0030 \\
\hline & & 3 & $\cdot$ & 0.7918 & 0.6230 & 0.5617 & 0.5301 & 0.1076 & 0.0254 & 0.0089 & 0,0035 \\
\hline & & 5 & - & 0.9918 & 0.7030 & 0,6017 & 0,5501 & 0.2643 & 0.0515 & 0.0155 & 0,0051 \\
\hline \multirow[t]{4}{*}{ Baves } & $\theta$ & $r$ & . & \multicolumn{8}{|c|}{ Inverse Chi-square distribution $(\mathbb{P},(\theta \mid x))$} \\
\hline & 0.5 & 2 & - & 0.54183 & 0.5230 & 0.5117 & 0.5051 & 0.0242 & 0,0108 & 0.0053 & 0.0027 \\
\hline & & 4 & . & 9257 & 029 & 0.5016 & 0.5001 & 0.0186 & 0.0095 & 0049 & 0.0026 \\
\hline & & 6 & & 0.45152 & 0.7030 & 0.4919 & 0.4952 & 0.0179 & 0.0515 & 0.0048 & 0,0025 \\
\hline \multirow[t]{5}{*}{ Bayes } & $\theta$ & $a$ & $B$ & \multicolumn{8}{|c|}{ Inverted Gamma distribution $\left(P_{d}(A \mid x)\right)$} \\
\hline & 0.5 & 2 & 0.5 & 0.4926 & 0,5029 & 0,5016 & 0,5001 & 0,0186 & 0.0095 & 0.0049 & 0,0026 \\
\hline & & 2 & l & 0.5380 & 0,5222 & 0.5114 & 0,5050 & 0.019 & 0.0099 & 0.0051 & 0,0026 \\
\hline & & 3 & 0.5 & 0.4515 & 0.4843 & 0.4919 & 0.4952 & 0.0179 & 0.0091 & 0.0048 & 0,0026 \\
\hline & & 3 & 1 & 0.4932 & 0,5028 & 0.5016 & 0,5001 & 0.0156 & 0.0088 & 0.0047 & 0.0025 \\
\hline \multirow[t]{10}{*}{ Bayes } & $\theta$ & $a$ & $\mathrm{~b}$ & \multicolumn{8}{|c|}{ Improper distribution $\left(\mathbb{P}_{1}(\theta \mid x)\right)$} \\
\hline & 0.5 & 1 & 1 & 0.4918 & 0.5430 & 0.5217 & 0.5101 & 0.0225 & 0,0121 & 0.0056 & 0,0027 \\
\hline & & 1 & 2 & 0.4471 & 0,5830 & 0.5417 & 0,5201 & 0.0213 & 0,0172 & 0.0069 & 0,0030 \\
\hline & & l & 3 & 0,7918 & 0.6230 & 0.5617 & 0.5301 & 0.1076 & 0.0254 & 0.0089 & 0,0035 \\
\hline & & 2 & 1 & 0.5380 & 0.5322 & 0.5114 & 0,5050 & 0.0199 & 0.0099 & 0.0047 & 0,0026 \\
\hline & & 2 & 2 & 0.6289 & 0.5606 & 0.5114 & 0.5149 & 0.0352 & 0.0132 & 0.0051 & 0,0028 \\
\hline & & 2 & 3 & 0,7198 & 0,5991 & 0.5506 & 0,5248 & 0.0669 & 0,0193 & 0.0075 & 0,0032 \\
\hline & & 3 & l & 0.4932 & 0.5028 & 0.5016 & 0,5001 & 0.0156 & 0.0088 & 0.0048 & 0,0025 \\
\hline & & 3 & 2 & 0,5765 & 0.3399 & 0,5208 & 0,5001 & 0.0214 & 0.0104 & 0.0052 & 0,0025 \\
\hline & & 3 & 3 & 0.6599 & 0.5769 & 0.5401 & 0.5001 & 0.0411 & 0.0147 & 0.0064 & 0,0025 \\
\hline Baves & $\theta$ & $c$ & . & \multicolumn{8}{|c|}{ Son-informative distribution $\left(\mathrm{P}_{c}(\theta \mid \mathrm{X})\right)$} \\
\hline & 0.5 & 1 & & 0.5465 & 0.524 & 0.5119 & 0.5051 & 0.0298 & 0.0117 & 0.0055 & 0.0027 \\
\hline & & 2 & . & 0,4918 & 0,5030 & 0,5017 & 0,5001 & 0.0225 & 0,0103 & 0,0052 & 0,0026 \\
\hline & & 3 & . & 0.4471 & 0.4837 & 0.4918 & 0.4951 & 0.0213 & 0,0098 & 0,0050 & 0,0026 \\
\hline
\end{tabular}

\begin{tabular}{|c|c|c|c|c|c|c|c|c|c|c|c|}
\hline \multirow{3}{*}{ Method } & \multirow{2}{*}{\multicolumn{3}{|c|}{ parameters }} & \multirow{2}{*}{\multicolumn{4}{|c|}{$\begin{array}{c}\wedge \\
\text { Estimate for } \theta(\theta)\end{array}$}} & \multicolumn{4}{|c|}{ MSE } \\
\hline & & & & & & & & \multicolumn{4}{|c|}{ Sample Size(n) } \\
\hline & $\theta$ & - & - & 10 & 25 & 50 & 100 & 10 & 25 & 50 & 100 \\
\hline ME & l & . & . & 1,0073 & 1,0074 & 0.9950 & 0.9984 & 0,1019 & 0.0400 & 0.0207 & 0.0100 \\
\hline IE & l & . & . & 1.0073 & 1,0074 & 0.9950 & 0.9984 & 1019 & 1.0400 & 0.0207 & 0.0100 \\
\hline \multirow[t]{4}{*}{ Baves } & $\theta$ & $b_{3}$ & . & \multicolumn{8}{|c|}{ Lerv distribution $(P,(\theta \mid x))$} \\
\hline & 1 & 0.5 & . & 10,323 & 25.435 & 50,003 & 100.1 & 97,112 & 622.08 & 2453.1 & 9920.5 \\
\hline & & 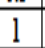 & . & 10.573 & 3.685 & 50.253 & 100,35 & 101.84 & 634,36 & 2477.7 & 9970.1 \\
\hline & & 2 & . & 11.073 & 26.185 & 50.753 & 100.85 & 111.66 & 659.29 & 2527.2 & 10070 \\
\hline Baves & $\theta$ & $\mathrm{b}$ & . & \multicolumn{8}{|c|}{ Gumbel trpe-II distribution $(P,(\theta \mid x))$} \\
\hline & 1 & 2 & . & 1,2073 & 1.0874 & 1,0351 & 1,0185 & 0.1448 & 0.0476 & 0.0219 & 0.0104 \\
\hline & & 3 & . & 1,3073 & 1.1274 & 1,0551 & 1.0285 & 0.1963 & 0.0562 & 0.0237 & 0.0108 \\
\hline & & 5 & . & 1,5073 & 1,2074 & 1,0951 & 1,0485 & 0.3592 & 0.0830 & 0.0297 & 0.0124 \\
\hline Baves & $\theta$ & $r$ & . & \multicolumn{8}{|c|}{ Inverse Chi-square distribution $(P,(\theta \mid x))$} \\
\hline & 1 & 2 & . & 1,0573 & 1.0274 & 1,0051 & 1.0035 & 0.1051 & 0.0407 & 0.0207 & 0.0100 \\
\hline & & 4 & . & 0.9612 & 0.9878 & 0.9853 & 0.9935 & 0,0857 & 0.0371 & 0.0201 & 0,0099 \\
\hline & & 6 & $\cdot$ & 0.8811 & 0.9512 & 0.9664 & 0.9837 & 0,0848 & 0.0366 & 0.0202 & 0,0099 \\
\hline Baves & $\theta$ & $a$ & $\beta$ & \multicolumn{8}{|c|}{ Inverted Gamma distribution $\left(P_{1}(\theta \mid x)\right)$} \\
\hline & 1 & 2 & 0.5 & 0.9612 & 0.9878 & 0.9853 & 0.9935 & 0.0857 & 0.0371 & 0.0201 & 0.0099 \\
\hline & & 2 & l & 1,0067 & 1,0071 & 0.9951 & 0.9984 & 0.0842 & 0.0370 & 0.0199 & 0,0098 \\
\hline & & 3 & 0.5 & 0.8811 & 0.9512 & 0.9664 & 0.9837 & 0.0848 & 0.0366 & 0.0202 & 0,0099 \\
\hline & & 3 & 1 & 0.9227 & 0.9698 & 0.9760 & 0.9886 & 0.0767 & 0.0352 & 0.0197 & 0.0097 \\
\hline Pon & $\theta$ & $a$ & $\mathrm{~b}$ & \multicolumn{8}{|c|}{ Improper distribution $\left(\mathrm{P}_{\imath}(\theta \mid \mathrm{X})\right)$} \\
\hline & 1 & 1 & 1 & 1,1073 & 1.0474 & 1,0151 & 1,0085 & 0.1134 & 0.0422 & 0.0209 & 0.0101 \\
\hline & & l & 2 & 1,2073 & 1,0874 & 1,0351 & 1,0185 & 0.1448 & 0.0476 & 0,0219 & 0,0104 \\
\hline & & 1 & 3 & 1,3073 & 1,1274 & 1.0551 & 1,0285 & 0.1963 & 0.0562 & 0.0237 & 0.0108 \\
\hline & & 2 & 1 & 1.0067 & 1.0071 & 0.9951 & 0.9984 & 0.0842 & 0.0370 & 0.0199 & 0,0098 \\
\hline & & 2 & 2 & 1.0976 & 1,0456 & 1,0148 & 1.0084 & 0.0937 & 0.0390 & 0.0201 & 0,0099 \\
\hline & & 2 & 3 & 1,1885 & 1.084 & 1,0344 & 1,0183 & 0.1197 & 0.0440 & 0.0211 & 0.0101 \\
\hline & & 3 & 1 & 0.9227 & 0.9698 & 0.9760 & 0.9886 & 0.0767 & 0.0352 & 0.0197 & 0.0097 \\
\hline & & 3 & 2 & 1.0061 & 1.0068 & 0.9952 & 0.9984 & 0.0707 & 0.0343 & 0.0191 & 0.0096 \\
\hline & & 3 & 3 & 1,0894 & 1.0439 & 1,0145 & 1.0083 & 0.0787 & 0.0362 & 0.0193 & 0,0097 \\
\hline$P_{1}$ & $\theta$ & $c$ & - & \multicolumn{8}{|c|}{ Non-informative distribution $\left(P_{c}(\theta \mid x)\right)$} \\
\hline & 1 & 1 & . & 1,1192 & 1.0494 & 1,0154 & 1.0085 & 0.1400 & 0.0458 & 0.0218 & 0.0103 \\
\hline & & 2 & . & 1.0073 & 1.0074 & 0.9950 & 0.9984 & 0.1019 & 0.0400 & 0.0207 & 0,0100 \\
\hline & & 3 & $\cdot$ & 0.9157 & 0.9686 & 0.9755 & 0.9885 & 0,0913 & 0.0379 & 0,0205 & 0,0099 \\
\hline
\end{tabular}




\section{Under Different Priors \&Two Loss Functions To Compare Bayes Estimators With Some of Classical Estimators For the Parameter of Exponential Distribution}

Table4.3: Shows the values for $\theta$ under square

square error loss function (MSE).

\begin{tabular}{|c|c|c|c|c|c|c|c|c|c|c|c|}
\hline \multirow{3}{*}{ Method } & \multirow{2}{*}{\multicolumn{3}{|c|}{ parameters }} & \multicolumn{4}{|c|}{ Estimate for $\theta(\theta)$} & \multicolumn{4}{|c|}{ MISE } \\
\hline & & & & \multicolumn{4}{|c|}{ Sample Size(n) } & \multicolumn{4}{|c|}{ Sample Size(n) } \\
\hline & $\theta$ & & & 10 & 25 & 50 & 100 & 10 & 25 & 50 & 100 \\
\hline ME & 2 & . & . & 2.0036 & 2,0018 & 2.0041 & 1.9954 & 0.39642 & 0.1607 & 0.0781 & \\
\hline IIE & 2 & & & 2.0036 & 2.0018 & 2.0041 & 1.99 & 0.39642 & 0.1607 & 0.0781 & \\
\hline \multirow[t]{4}{*}{ Baves } & $\theta$ & $b_{3}$ & . & \multicolumn{8}{|c|}{ Lerv distribution $(P,(\theta \mid x))$} \\
\hline & 2 & 0.5 & . & 20.286 & 50.296 & 100.46 & 199.79 & 374,02 & 2432.9 & 9888.7 & 39529 \\
\hline & & 1 & & 20.536 & 50.546 & 100,71 & 200.04 & & 2457,1 & & \\
\hline & & 2 & - & 21.036 & 51,046 & 101.21 & 200.54 & 402.01 & 505.9 & 10037 & \\
\hline aves & $\theta$ & $b$ & & \multicolumn{8}{|c|}{ Gumbel trpe-Idistribution $\left(P_{1}(\theta \mid x)\right)$} \\
\hline & 2 & 2 & . & 2.2036 & 2.0818 & 2,0441 & 20154 & 0.4378 & .1674 & 0.0800 & 0.0411 \\
\hline & & 3 & . & 2,3036 & 2.1218 & 2.0641 & 2.0254 & 0.4885 & 0.1755 & 0.0822 & 0.0415 \\
\hline & & 5 & & 2,5036 & 2.2018 & 2.1041 & 2,0454 & 0.6500 & 0.2014 & 0,0889 & 0.0429 \\
\hline Baves & $\theta$ & $r$ & . & \multicolumn{8}{|c|}{ Inverse Chi-square distribution $(P,(\theta \backslash x))$} \\
\hline & 2 & 2 &. & 2.0536 & 2.0218 & 2,0141 & 2.0004 & 0.3992 & 0.1611 & 0.0783 & 0.0408 \\
\hline & & 4 & . & 1.8669 & 1,9441 & 1,9746 & 1.9806 & 0,3453 & 0,1517 & 0.0757 & 0.0404 \\
\hline & & 6 & & 1,7113 & 1.8721 & 1,9366 & 1,9612 & 0.3586 & 0.1541 & 0.0762 & 0.0407 \\
\hline Baves & $\theta$ & $a$ & B & \multicolumn{8}{|c|}{ Inverted Gamma distribution $\left(\mathrm{P}_{1}(\theta \mid \mathrm{X})\right)$} \\
\hline & 2 & 2 & 0.5 & 1.8669 & 1.9441 & 1,9746 & 1,9806 & 0.3453 & 0.1517 & 0.0757 & 0.0404 \\
\hline & & 2 & 1 & 1.9124 & 1.9633 & 1.9844 & 1.9855 & 0,3352 & 0.1499 & 0.0753 & 0.0402 \\
\hline & & 3 & 0.5 & 1,7113 & 1.8721 & 1,9366 & 1.9612 & 0.3586 & 0.1541 & 0.0762 & 0.0407 \\
\hline & & 3 & 1 & 1,753 & 1.8906 & 1,9463 & 1.9661 & 0.3362 & 0.1497 & 0.0751 & 0.0404 \\
\hline Bayes & $\theta$ & $a$ & $\mathrm{~b}$ & \multicolumn{8}{|c|}{ Improper distribution $\left(P_{2}(\theta \mid X)\right)$} \\
\hline & 2 & 1 & 1 & 2.1036 & 2.0418 & 2.0241 & 2.0054 & 0.4071 & 0.1624 & 0.0786 & 0.0409 \\
\hline & & 1 & 2 & 2.2036 & 2.0818 & 2.0441 & 2.0154 & 0.4378 & 0.1674 & 0.0800 & 0.0411 \\
\hline & & 1 & 3 & 2,3036 & 2.1218 & 2.0641 & 2.0254 & 0.4885 & 0.1755 & 0,0822 & 0.0415 \\
\hline & & 2 & 1 & 1.9124 & 1.9633 & 1.9844 & 1.9855 & 0,3352 & 0.1499 & 0.0753 & 0.0402 \\
\hline & & 2 & 2 & 2.0033 & 2.0018 & 2.004 & 1.9954 & 0,3276 & 0.1485 & 0.0750 & 0,0400 \\
\hline & & 2 & 3 & 2.0942 & 2.0402 & 2.0236 & 2.0053 & 0,3364 & 0.1502 & 0.0756 & 0,0401 \\
\hline & & 3 & 1 & 1,753 & 1.8906 & 1,9463 & 1.9661 & 0,3362 & 0.1497 & 0.0751 & 0.0404 \\
\hline & & 3 & 2 & 1.8363 & 1.9276 & 1.9655 & 1.9759 & 0,3020 & 0.1430 & 0.0734 & 0.0398 \\
\hline & & 3 & 3 & 1.9197 & 1.9647 & 1,9847 & 1,9857 & 0.2817 & 0.1390 & 0.0724 & 0.0394 \\
\hline Baves & $\theta$ & $c$ & . & \multicolumn{8}{|c|}{ Non-informative distribution $\left.\left(\mathrm{P}_{6}(\theta) \mathrm{x}\right)\right)$} \\
\hline & 2 & 1 & . & 2.2262 & 2.0852 & 2,045 & 2.0155 & 0.5405 & 0.1816 & 0.0833 & 0.0419 \\
\hline & & 2 & . & 2.0036 & 2.0018 & 2,0041 & 1.9954 & 0,3964 & 0.1607 & 0,0781 & 0,0409 \\
\hline & & 3 & & 1.8215 & 1.9248 & 1.9648 & 1.9756 & 0.3594 & 0.1542 & 0.0763 & 0.0406 \\
\hline
\end{tabular}

Table 4.4: Shows the values for $\theta$ under

error loss function (MSE).

\begin{tabular}{|c|c|c|c|c|c|c|c|c|c|c|c|}
\hline \multirow{3}{*}{ Method } & \multirow{2}{*}{\multicolumn{3}{|c|}{ parameters }} & \multirow{2}{*}{\multicolumn{4}{|c|}{$\frac{\text { Estimate for } \theta(\theta)}{\text { Sample Size(n) }}$}} & \multicolumn{4}{|c|}{ MSE } \\
\hline & & & & & & & & \multicolumn{4}{|c|}{ Sample Size(n) } \\
\hline & $\theta$ & . & . & 10 & 25 & 50 & 100 & 10 & 25 & 50 & 100 \\
\hline ME & 4 & . & . & 4.0072 & 4.0037 & 4,0133 & 3.998 & 1.5857 & 0.6428 & 0,3301 & 0.1581 \\
\hline IIE & 4 & . & - & 4.0072 & 4.0037 & 4,0133 & 3.998 & 1.5857 & 0.6428 & 0.3301 & 0.1581 \\
\hline \multirow[t]{4}{*}{ Bayes } & $\theta$ & $b_{3}$ & - & \multicolumn{8}{|c|}{ Lerv distribution $(P,(\theta \mid x))$} \\
\hline & 4 & 0.5 & . & 40,322 & 100.34 & 200.91 & 400,05 & 1477.9 & 9683.4 & 39600 & $1.584 \mathrm{e}^{+5}$ \\
\hline & & & - & 40.572 & 100.59 & 201.16 & 400,3 & 1496.1 & 9731.6 & 39698 & $1,586 \mathrm{e}^{+5}$ \\
\hline & & 2 & . & 41.072 & 101.09 & 201.66 & 400.8 & 1532.9 & 9828.5 & 39896 & $1.590 e^{+5}$ \\
\hline Baves & $\theta$ & $\mathrm{b}$ & . & \multicolumn{8}{|c|}{ Gumbel trpe-IIdistribution $\left(P_{1}(\theta \mid x)\right)$} \\
\hline & 4 & 2 & . & 4.2072 & 4.0837 & 4,0533 & 4.018 & 1.6286 & 0.6498 & 0.3328 & 0.1584 \\
\hline & & 3 & . & 4,3072 & 4.1237 & 4.0733 & 4.028 & 1.68 & 0.6581 & 0.3353 & 0.1589 \\
\hline & & 5 & - & 4.5072 & 4.2037 & 4,1133 & 4.048 & 1.8429 & 0.6842 & 0.3428 & 0.1604 \\
\hline Baves & $\theta$ & $r$ & . & \multicolumn{8}{|c|}{ Inverse Chi-square distribution $(\mathrm{P},(\mathrm{O} \mid \mathrm{X}))$} \\
\hline & 4 & 2 & . & 4.0572 & 4.0237 & 4,0233 & 4.003 & 1.5889 & 0.6433 & 0.3305 & 0.1581 \\
\hline & & 4 & - & 3.6884 & 3.8689 & 3.9444 & 3.9634 & 1.4076 & 0.6115 & 0.3203 & 0.1563 \\
\hline & & 6 & . & 3,381 & 3,7256 & 3.8685 & 3.9245 & 1.4843 & 0.6264 & 0.3224 & 0.1576 \\
\hline Baves & $\theta$ & $a$ & B & \multicolumn{8}{|c|}{ Inverted Gamma distribution $\left(P_{1}(\theta \mid X)\right)$} \\
\hline & 4 & 2 & 0.5 & 3.6884 & 3.8689 & 3.9444 & 3.9634 & 1.4076 & 0.6115 & 0.3203 & 0.1563 \\
\hline & & 2 & 1 & 3,7338 & 3.8881 & 3.9542 & 3.9683 & 1,3813 & 0.6068 & 0.3193 & 0.1560 \\
\hline & & 3 & 0.5 & 3,381 & 3,7256 & 3.8685 & 3.9245 & 1.4843 & 0.6264 & 0.3224 & 0.1576 \\
\hline & & 3 & 1 & 3.4227 & 3.7441 & 3.8781 & 3.9294 & 1.4345 & 0.6165 & 0.3199 & 0.1569 \\
\hline Baves & $\theta$ & a & $\mathrm{b}$ & \multicolumn{8}{|c|}{ Improper distribution $\left(\mathrm{P}_{1}(\theta \mid \mathrm{x})\right)$} \\
\hline & 4 & 1 & 1 & 4,1072 & 4.0437 & 4,0333 & 4.008 & 1.5971 & 0.6447 & 0.3311 & 0.1582 \\
\hline & & 1 & 2 & 4.2072 & 4.0837 & 4.0533 & 4.018 & 1.6286 & 0.6498 & 0.3328 & 0.1584 \\
\hline & & 1 & 3 & 4,3072 & 4.1237 & 4.0733 & 4.028 & 1.68 & 0.6581 & 0.3353 & 0.1589 \\
\hline & & 2 & l & 3,7338 & 3.8881 & 3.9542 & 3.9683 & 1,3813 & 0.6068 & 0.3193 & 0.1560 \\
\hline & & 2 & 2 & 3.8247 & 3.9266 & 3.9738 & 3.9782 & 1.3412 & 0.5997 & 0.3178 & 0.1554 \\
\hline & & 2 & 3 & 3.9156 & 3.9651 & 3.9934 & 3.9881 & 1,3176 & 0,5955 & 0,3172 & 0.1551 \\
\hline & & 3 & l & 3.4227 & 3.7441 & 3.8781 & 3.9294 & 1.4345 & 0.6165 & 0,3199 & 0.1569 \\
\hline & & 3 & 2 & 3,506 & 3,7812 & 3.8974 & 3.9392 & 1,3452 & 0.599 & 0,3156 & 0.1556 \\
\hline & & 3 & 3 & 3,5893 & 3.8182 & 3.9166 & 3.949 & 1.2698 & 0.5841 & 0,3120 & 0.1545 \\
\hline Baves & $\theta$ & $\mathrm{c}$ & . & \multicolumn{8}{|c|}{ Son-informative distribution $\left(P_{c}(\theta \mid x)\right)$} \\
\hline & 4 & 1 & . & 4.4524 & 4.1705 & 4,0952 & 4.0384 & 2.1623 & 0.7265 & 0.3526 & 0.1628 \\
\hline & & 2 & . & 4.0072 & 4.0037 & 4.0133 & 3.998 & 1.5857 & 0.6428 & 0.3301 & 0.1581 \\
\hline & & 3 & . & 3.6429 & 3.8497 & 3.9346 & 3.9584 & 1.438 & 0.6169 & 0.3214 & 0.1567 \\
\hline
\end{tabular}




\section{Under Different Priors \&Two Loss Functions To Compare Bayes}

In table (4.5), we obtained over estimated values for $\theta(\theta)$, when the prior distribution for $\theta$ is Gumbel type-II distribution, for all assuming values for $b$, when the true value of $\theta(\theta=0.5)$, and for all samples sizes (n) comparative to the estimated values by using MLE and ME according to the smallest values of MWSE for all samples sizes (n).

But in general, we obtained a good estimation according to the smallest values of MWSE for all samples sizes (n) comparative to the estimated values by using MLE and ME .we listed them when the prior distribution for $\theta$ are

- Levy distribution with $b_{3}=0.5$.

- Inverse Chi-square distribution with $v=4$.

- Inverted Gamma distribution with $(\alpha=3, \beta=1)$.

- Improper distribution with $(a=3, b=1)$.

- Non-informative distribution with $\mathrm{c}=2$.

In table (4.6), when the true value of $\theta(\theta=1)$ in general, we obtained a good estimation according to the smallest values of MWSE for all samples sizes (n) comparative to the estimated values by using MLE and ME .we listed them when the prior distribution for $\theta$ are

- Levy distribution with $b_{3}=1$.

- Gumbel type-II distribution with $b=2$.

- Inverse Chi-square distribution with $v=4$.

- Inverted Gamma distribution with $(\alpha=2, \beta=1)$.

- Improper distribution with $(\mathrm{a}=\mathrm{b}=3)$.

- Non-informative distribution with $\mathrm{c}=2$.

In table (4.7), when the true value of $\theta(\theta=2)$ in general, we obtained a good estimation according to the smallest values of MWSE for all samples sizes (n) ,comparative to the estimated values by using MLE and ME .we listed them when the prior distribution for $\theta$ are

- Levy distribution with $b_{3}=2$.

- Gumbel type-II distribution with $b=2$.

- Inverse Chi-square distribution with $\mathbf{v}=2$.

- Inverted Gamma distribution with $(\alpha=2, \beta=1)$.

- Improper distribution with $(a=2, b=3)$.

- Non-informative distribution with $\mathrm{c}=2$. 


\section{Under Different Priors \& Two Loss Functions To Compare Bayes}

In table (4.8), when the true value of $\theta(\theta=4)$ in general, we obtained a good estimation according to the smallest values of MWSE for all samples sizes (n) ,comparative to the estimated values by using MLE and ME .we listed them when the prior distribution for $\theta$ are

- Levy distribution with $b_{3}=2$.

- Gumbel type-II distribution with $b=3$.

- Inverse Chi-square distribution with $v=2$.

- Inverted Gamma distribution with $(\alpha=2, \beta=1)$.

- Improper distribution with $(a=1, b=3)$.

- Non-informative distribution with $\mathrm{c}=2$. 


\section{Under Different Priors \&Two Loss Functions To Compare Bayes Estimators With Some of Classical Estimators For the Parameter of Exponential Distribution}

Table 4.5: Shows the values for $\theta$ under weighted weighted

square error loss function (MWSE).

\begin{tabular}{|c|c|c|c|c|c|c|c|c|c|c|c|}
\hline \multirow{3}{*}{ Method } & \multirow{2}{*}{\multicolumn{3}{|c|}{ parameters }} & \multicolumn{4}{|c|}{$\begin{array}{c}\lambda \\
\text { Estimate for } \theta(\theta)\end{array}$} & \multicolumn{4}{|c|}{ MTSE } \\
\hline & & & & \multicolumn{4}{|c|}{ Sample Size(n) } & \multicolumn{4}{|c|}{ Sample Size(n) } \\
\hline & $\theta$ & " & - & 10 & 25 & 50 & 100 & 10 & 25 & 50 & 100 \\
\hline MLE & & & & 0.4918 & 0.5030 & 0.5017 & 0,5001 & 0.04497 & 0.0206 & 0.0103 & 0.0053 \\
\hline IIE & & & & & & 0.5017 & 0,5001 & & 9006 & 0103 & \\
\hline \multirow[t]{4}{*}{ Bayes } & $\theta$ & $b_{3}$ & - & \multicolumn{8}{|c|}{ Lery distribution $\left(P_{1}(\theta \mid x)\right)$} \\
\hline & 0.5 & 0.5 & & 0.4922 & 0,5029 & 0,5016 & 0,5001 & 0.0407 & 0,0197 & 0,0101 & 0.0052 \\
\hline & & & & & & 0.5065 & & & & & \\
\hline & & & & 0.5636 & 0.5323 & 0.5164 & 0.5075 & 0.0487 & & 0.0106 & \\
\hline Bayes & $\theta$ & $\mathrm{b}$ & & \multicolumn{8}{|c|}{ Gumbeltype-Id distribution $(\mathrm{P},(\mathrm{\theta} \mid \mathrm{x}))$} \\
\hline & 0.5 & 2 & & 0.6289 & 0.5606 & 0.5310 & 0.5149 & 0.0703 & 0263 & & 0.0056 \\
\hline & & 3 & - & 0.7198 & 0.5991 & 0.5506 & 0.5248 & 0.1337 & 0.0386 & 0.0150 & 0.0063 \\
\hline & & 5 & . & 0.9016 & 0.676 & 0.5898 & 0.5446 & 0,3597 & 0,0809 & 0.0260 & 0.0091 \\
\hline Baves & $\theta$ & $r$ & - & \multicolumn{8}{|c|}{ Inverse Chi-square distribution $(P,(\theta \mid x))$} \\
\hline & 0.5 & 2 & $\dot{.}$ & 0.4926 & 0,5029 & 0,5016 & 0,5001 & 0.0372 & 0,0190 & 0.0099 & 0.0051 \\
\hline & & 4 & . & 0.4515 & 0.4843 & 0.4919 & 0.4951 & 0.0358 & 0,0181 & 0.0096 & 0.0051 \\
\hline & & 6 & & 0.4167 & 0.467 & 0.4827 & 0.4903 & 0.0403 & 0.0185 & 0.0097 & 0.0051 \\
\hline Bayes & $\theta$ & $a$ & $\beta$ & \multicolumn{8}{|c|}{ Inverted Gamma distribution $\left(\mathrm{P}_{i}(\mathrm{O} \mid \mathrm{X})\right)$} \\
\hline & 0.5 & 2 & 0.5 & 0.4515 & 0.4843 & 0.4919 & 0.4951 & 0.0358 & 0.0181 & 0.0096 & 0.0051 \\
\hline & & 2 & l & 0.4932 & 0.5028 & 0.5015 & 0,5001 & 0.0312 & 0.0176 & 0.0095 & 0.0050 \\
\hline & & 3 & 0.5 & 0.4167 & 0.467 & 0.4827 & 0.4903 & 0,0403 & 0.0185 & 0.0097 & 0.0051 \\
\hline & & 3 & 1 & 0.4552 & 0.4848 & 0.4921 & 0.4952 & 0.0305 & 0.0168 & 0.0093 & 0.0050 \\
\hline Baves & $\theta$ & $a$ & $\mathrm{~b}$ & \multicolumn{8}{|c|}{ Improper distribution $(P,(\theta \mid x))$} \\
\hline & 0.5 & l & l & 0.5380 & 0,5221 & 0.5114 & 0.5050 & 0.0399 & 0.0199 & 0.0101 & 0.0052 \\
\hline & & l & 2 & 0.6289 & 0.5606 & 0.5310 & 0.5149 & 0,0703 & 0.0263 & 0,0118 & 0.0056 \\
\hline & & 1 & 3 & 0.7198 & 0,5990 & 0.5506 & 0.0051 & 0.1337 & 0.0386 & 0.0150 & 0.0063 \\
\hline & & 2 & l & 0.4931 & 0.5028 & 0.5015 & 0.5001 & 0,0312 & 0,0176 & 0,0095 & 0.0051 \\
\hline & & 2 & 2 & 0.5765 & 0.5398 & 0.5208 & 0.5098 & 0.0428 & 0.0207 & 0.0104 & 0.0052 \\
\hline & & 2 & 3 & 0.6598 & 0,5768 & 0.5400 & 0.5196 & 0.0822 & 0.0294 & 0.0127 & 0.0058 \\
\hline & & 3 & & 0.4552 & 0.4848 & 0.4921 & 0.4952 & 0.0305 & 0,0168 & 0.0093 & 0,0050 \\
\hline & & 3 & 2 & 0.5321 & 0.5205 & 0.511 & 0.5049 & 0.0286 & 0.0172 & 0.0094 & 0.0050 \\
\hline & & 3 & 3 & 0.6091 & 0.5562 & 0,5298 & 0.5146 & 0.0503 & 0.0227 & 0,0109 & 0.0053 \\
\hline Bayes & $\theta$ & c & & \multicolumn{8}{|c|}{ Non-informative distribution $\left(\mathrm{P}_{k}(\theta \mid \mathrm{X})\right)$} \\
\hline & 0.5 & l & . & 0.4918 & 0,5030 & 0,5016 & 0,5000 & 0.0449 & 0.0205 & 0.0103 & 0,0052 \\
\hline & & 2 & - & 0.4471 & 0.4836 & 0.4918 & 0.4951 & 0.0426 & 0.0195 & 0.0100 & 0,0052 \\
\hline & & 3 & & 0.4098 & 0.4657 & 0.4823 & 0.4902 & 0.0473 & 0.0199 & 0.0101 & 0.0052 \\
\hline
\end{tabular}

Table 4.6: Shows the values for $\theta$ under

square error loss function (MWSE).

\begin{tabular}{|c|c|c|c|c|c|c|c|c|c|c|c|}
\hline \multirow{3}{*}{ Method } & \multirow{2}{*}{\multicolumn{3}{|c|}{ parameters }} & \multicolumn{4}{|c|}{ Estimate for $\theta(\theta)$} & \multicolumn{4}{|c|}{ MITSE } \\
\hline & & & & \multicolumn{4}{|c|}{ Sample Sire(n) } & \multicolumn{4}{|c|}{ Sample Size(n) } \\
\hline & $\theta$ & & & 10 & 25 & 50 & 100 & 10 & 25 & 50 & 100 \\
\hline .LE & $\mathrm{l}$ & & & 1.0073 & 1.0074 & 0.9950 & 0.9984 & 0.1019 & 0.0400 & 0.0207 & \\
\hline IE & & & & & 1.0074 & 0.9950 & 0.9984 & & & & \\
\hline \multirow[t]{4}{*}{ Bayes } & $\theta$ & $b_{3}$ & & \multicolumn{8}{|c|}{ Lery distribution $\left(P_{1}(\theta \mid x)\right)$} \\
\hline & & 0.5 & & 0.9831 & 0.9974 & 0.9901 & 0.9959 & & 0.0384 & 0.0204 & \\
\hline & & & & & & & & & & & \\
\hline & & 2 & & 1.0546 & 1.0269 & 1.005 & 1,0034 & 0953 & 0.0391 & 20203 & 0.009 \\
\hline \multirow[t]{4}{*}{ Bayes } & $\theta$ & $\mathrm{b}$ & & \multicolumn{8}{|c|}{ Gumbel type-Idistribution $(P,(\theta \mid x))$} \\
\hline & & 2 & & 1.0976 & 1.0456 & 1.0148 & 1.0084 & 937 & 390 & 0201 & 0.0099 \\
\hline & & 3 & . & & 1.084 & 1.0344 & 1.0183 & 197 & 0.0440 & 0211 & 0.0101 \\
\hline & & 5 & & & 1.161 & 1.0736 & 1.0381 & 213 & 0.0629 & 0253 & 0.011. \\
\hline \multirow[t]{4}{*}{ Baves } & $\theta$ & $\mathrm{r}$ & & \multicolumn{8}{|c|}{ Inverse Chi-squaredistribution $(\mathrm{P},(\theta \mid \mathrm{X}))$} \\
\hline & 1 & 2 & & 0.9612 & 0.9878 & 0.9853 & 0.9935 & 0.0857 & 0.0371 & 0201 & 0.0099 \\
\hline & & 4 & & & & 664 & 0.9837 & & 0.0366 & 20202 & 0.0099 \\
\hline & & 6 & & 0.8133 & 0.9173 & 0.9481 & 0.9742 & 0951 & 0.03874 & 0211 & 0.0101 \\
\hline \multirow[t]{5}{*}{ Bayes } & $\theta$ & $a$ & $\beta$ & \multicolumn{8}{|c|}{ Inverted Gamma distribution $\left(P_{4}(\theta \mid x)\right)$} \\
\hline & 1 & 2 & 0.5 & 0.8811 & 0,9512 & $0.966 t$ & 0.9837 & 0.0848 & 0.0366 & 0.0202 & 0.0099 \\
\hline & & 2 & & & & 760 & 886 & 767 & 352 & 197 & 0.0097 \\
\hline & & 3 & 0.5 & 0.8133 & 0.9173 & 0.9481 & 0.9742 & 0.0951 & 0.0387 & 0.0211 & 0.010 \\
\hline & & 3 & 1 & 0.8517 & 0.9351 & 0.9576 & 0.9790 & 0.0822 & 361 & 0.0202 & 0.0099 \\
\hline \multirow[t]{10}{*}{ Baves } & $\theta$ & a & b & \multicolumn{8}{|c|}{ Improperdistribution $\left.\left(P_{1}(\theta) \times\right)\right)$} \\
\hline & 1 & 1 & $\mathrm{l}$ & & 1.0071 & 0.9951 & 0.9984 & & 0.0370 & 0199 & 0.0098 \\
\hline & & $\mathrm{l}$ & 7 & & & & & & & 201 & 0.0099 \\
\hline & & l & 3 & & 1.084 & 1.0344 & 1.0183 & 197 & 440 & 2011 & 0.010 \\
\hline & & 2 & & & 0.9698 & 0.9760 & 0.9886 & 0.0767 & 352 & 0.0197 & 0.0097 \\
\hline & & 2 & 2 & & 1.0068 & 0.9952 & 0.9 & 0.0707 & 343 & 0.0191 & 0.0096 \\
\hline & & 2 & 3 & & 1.0439 & 1.0145 & 1.0083 & 0.0787 & 0.0362 & 0193 & 0.0097 \\
\hline & & 1 & & & 0.9351 & 0.9576 & 0.9790 & & & 0202 & 0.0099 \\
\hline & & 3 & 2 & 0.9287 & 0.9708 & 0.9764 & 0.9887 & 353 & 327 & 0.019 & 0.0096 \\
\hline & & 3 & 3 & 1.0056 & 1.0066 & 0.9953 & 0.9985 & 0.0603 & 0.0319 & 0.0184 & 0.0094 \\
\hline & $\theta$ & c & & \multicolumn{4}{|c|}{ Mon-Informative distribution $\quad\left(P_{k}(\theta)\right.$} & & & & \\
\hline & 1 & 1 & & & 1.0074 & 0.9950 & 0.9984 & 0.1019 & & 0207 & 0.0100 \\
\hline & & 2 & & 0.9157 & 0.9686 & 0.9755 & 0.9885 & 0.0913 & 0.0379 & 0.0205 & 0.0099 \\
\hline & & 3 & & & 0.9327 & 0.9567 & 0.9788 & 0.0963 & 0.0388 & 0.0210 & \\
\hline
\end{tabular}




\section{Under Different Priors \&Two Loss Functions To Compare Bayes Estimators With Some of Classical Estimators For the Parameter of Exponential Distribution}

Table4.7: Shows the values for $\theta$ under weighted weighted

square error loss function (MWSE).
Table4.8: Shows the values for $\theta$ under

square error loss function (MWSE).

\begin{tabular}{|c|c|c|c|c|c|c|c|c|c|c|c|}
\hline \multirow{3}{*}{ Method } & \multirow{2}{*}{\multicolumn{3}{|c|}{ parameters }} & \multicolumn{4}{|c|}{ Estimate for $\theta(\theta)$} & \multicolumn{4}{|c|}{ MISE } \\
\hline & & & & \multicolumn{4}{|c|}{ Sample Size(n) } & \multicolumn{4}{|c|}{ Sample Size(n) } \\
\hline & $\theta$ & . & . & 10 & 25 & 50 & 100 & 10 & 25 & 50 & 100 \\
\hline MLE & 2 & . & $\dot{-}$ & 2.0036 & 2.0018 & 2.0041 & 1.9954 & 0.1982 & 0.0803 & 0.0390 & 0.0204 \\
\hline .IE & 2 & . & . & 2.0036 & 2.0018 & 2.0041 & 1.9954 & 0.1982 & 0.0803 & 0.0390 & 0.0204 \\
\hline Bayes & $\theta$ & $b_{3}$ & . & \multicolumn{8}{|c|}{ Lery distribution $\left(P_{1}(\theta \mid x)\right)$} \\
\hline & 2 & 0.5 & . & 1.932 & 1.9724 & 1.9892 & 1.988 & 0.1820 & 0.0776 & 0.0383 & 0.0203 \\
\hline & & l & . & 1.9558 & 1.9822 & 1.9942 & 1.9904 & 0.1807 & 0.0773 & 0.0383 & 0.0202 \\
\hline & & 2 & - & 2.0034 & 2.0018 & 2.0041 & 1.9954 & 0.1797 & 0.0772 & 0.0382 & 0.0202 \\
\hline Bayes & $\theta$ & $\mathrm{b}$ & - & \multicolumn{8}{|c|}{ Gumbel type-II distribution $\left(P_{1}(\theta \mid x)\right)$} \\
\hline & 2 & 2 & . & 2.0033 & 2.0018 & 2.004 & 1.9954 & 0.1638 & 0.0742 & 0.0375 & 0.0200 \\
\hline & & 3 & $\cdot$ & 2.0942 & 2.0402 & 2.0236 & 2.0053 & 0.1682 & 0.0750 & 0.0378 & 0.0200 \\
\hline & & 5 & . & 2.276 & 2.1171 & 2.0628 & 2.0251 & 0.2018 & 0.0811 & 0.0395 & 0.0203 \\
\hline Baves & $\theta$ & $\mathrm{v}$ & . & \multicolumn{8}{|c|}{ Inverse Chi-square distribution $(P, \theta \mid x)$} \\
\hline & 2 & 2 & . & 1.8669 & 1.9441 & 1.9746 & 1.9806 & 0.1726 & 0.0758 & 0.0378 & 0.0202 \\
\hline & & 4 & . & 1,7113 & 1.8721 & 1.9366 & 1.9612 & 0.1793 & 0.0770 & 0.0381 & 0.0204 \\
\hline & & 6 & . & 1,5797 & 1.8052 & 1.9001 & 1.9421 & 0.2056 & 0.0830 & 0.0397 & 0.0209 \\
\hline Bayes & $\theta$ & $a$ & $\beta$ & \multicolumn{8}{|c|}{ Inverted Gamma distribution $\left(\mathrm{P}_{d}(\theta \mid \mathrm{X})\right)$} \\
\hline & 2 & 2 & 0.5 & 1,7113 & 1.8721 & 1.9366 & 1.9612 & 0.1793 & 0.0770 & 0.0381 & 0.0204 \\
\hline & & 2 & l & 1.753 & 1.8906 & 1.9463 & 1.9661 & 0.1681 & 0.0748 & 0.0375 & 0.0202 \\
\hline & & 3 & 0.5 & 1.5797 & 1.8052 & 1.9001 & 1.9421 & 0.2056 & 0.0830 & 0.0397 & 0.0209 \\
\hline & & 3 & l & 1.6182 & 1.8231 & 1.9095 & 1.947 & 0.1901 & 0.0797 & 0.0388 & 0.0206 \\
\hline Bares & $\theta$ & $a$ & $\mathrm{~b}$ & \multicolumn{8}{|c|}{ Improperdistribution $\left(\mathrm{P}_{c}(\theta \mid \mathrm{x})\right)$} \\
\hline & 2 & 1 & 1 & 1.9124 & 1.9633 & 1.9844 & 1.9855 & 0.1676 & 0.0749 & 0.0376 & 0.0201 \\
\hline & & l & 2 & 2.0033 & 2.0018 & 2.004 & 1.9954 & 0.1638 & 0.0742 & 0.0375 & 0.0200 \\
\hline & & l & 3 & 2.0942 & 2.0402 & 2.0236 & 2.0053 & 0.1682 & 0.0750 & 0.0378 & 0.0200 \\
\hline & & 2 & l & 1.753 & 1.8906 & 1.9463 & 1.9661 & 0.1681 & 0.0748 & 0.0375 & 0.0202 \\
\hline & & 2 & 2 & 1.8363 & 1.9276 & 1.9655 & 1.9759 & 0.1510 & 0.0715 & 0.0367 & 0.0199 \\
\hline & & 2 & 3 & 1.9197 & 1.9647 & 1.9847 & 1.9857 & 0.1408 & 0.0695 & 0.0362 & 0.0197 \\
\hline & & 3 & $\mathrm{l}$ & 1,6182 & 1.8231 & 1,9095 & 1.947 & 0.1901 & 0.0797 & 0.0388 & 0.0206 \\
\hline & & 3 & 2 & 1.6951 & 1.8588 & 1.9284 & 1.9567 & 0.1637 & 0.0740 & 0.0373 & 0.0202 \\
\hline & & 3 & 3 & 1,772 & 1.8945 & 1.9473 & 1.9664 & 0.1432 & 0.0696 & 0.0361 & 0.0198 \\
\hline Bayes & $\theta$ & $\mathrm{c}$ & & \multicolumn{8}{|c|}{ Non-informative distribution $\left(\mathrm{P}_{k}(\theta \mid \mathrm{X})\right)$} \\
\hline & 2 & 1 & . & 2.0036 & 2.0018 & 2.0041 & 1.9954 & 0.1982 & 0.0803 & 0,0390 & 0.0204 \\
\hline & & 2 & . & 1.8215 & 1.9248 & 1.9648 & 1.9756 & 0.1797 & 0.0771 & 0.0381 & 0.0203 \\
\hline & & 3 & . & 1.6699 & 1.8535 & 1.927 & 1.9563 & 0.1922 & 0.0796 & 0.0387 & 0.0206 \\
\hline
\end{tabular}

\begin{tabular}{|c|c|c|c|c|c|c|c|c|c|c|c|}
\hline \multirow{3}{*}{ Method } & \multirow{2}{*}{\multicolumn{3}{|c|}{ parameters }} & \multirow{2}{*}{\multicolumn{4}{|c|}{$\frac{\text { Estimate for } \theta(\theta)}{\text { Sample Size }(n)}$}} & \multicolumn{4}{|c|}{ MWSE } \\
\hline & & & & & & & & \multicolumn{4}{|c|}{ Sample Size(n) } \\
\hline & $\theta$ & . & . & 10 & 25 & 50 & 100 & 10 & 25 & 50 & 100 \\
\hline ME & 4 & . & . & 4.0072 & 4.0037 & 4.0133 & 3.998 & 0.3964 & 0.1607 & 0.0825 & 0.0395 \\
\hline IIE & 4 & . & - & 4.0072 & 4.0037 & 4.0133 & 3.998 & 0.3964 & 0.1607 & 0,0825 & 0,0395 \\
\hline \multirow[t]{4}{*}{ Bayes } & $\theta$ & $b_{3}$ & - & \multicolumn{8}{|c|}{ Lery distribution $\left(\mathrm{P}_{1}(\theta \mid \mathrm{x})\right)$} \\
\hline & 4 & 0.5 & . & 3.8402 & 3.935 & 3.9785 & 3.9806 & 0.3659 & 0.1555 & 0.0809 & 0.0392 \\
\hline & & l & $\dot{.}$ & 3.864 & 3.9448 & 3.9834 & 3.9831 & 0.3641 & 0.1552 & 0.0809 & 0.0392 \\
\hline & & 2 & - & 3.9116 & 3.9644 & 3.9933 & 3.9881 & 0.3615 & 0.1547 & 0.0808 & 0.0391 \\
\hline Bayes & $\theta$ & $\mathrm{b}$ & $\dot{-}$ & \multicolumn{8}{|c|}{ Gumbel type-II distribution $(\mathrm{P},(\theta \mid \mathrm{X}))$} \\
\hline & 4 & 2 & . & 3.8247 & 3.9266 & 3.9738 & 3.9782 & 0.3352 & 0.1499 & 0.0794 & 0.0388 \\
\hline & & 3 & - & 3.9156 & 3.9651 & 3.9934 & 3.9881 & 0.3293 & 0.1488 & 0.0793 & 0.0387 \\
\hline & & 5 & $\dot{.}$ & 4.0975 & 4,042 & 4.0326 & 4.0079 & 0.3299 & 0.1490 & 0.0795 & 0.0387 \\
\hline Baves & $\theta$ & $r$ & . & \multicolumn{8}{|c|}{ Inverse Chi-square distribution $(\mathrm{P},(\theta \mid \mathrm{X}))$} \\
\hline & 4 & 2 & . & 3.6884 & 3.8689 & 3.9444 & 3.9634 & 0.3518 & 0.1528 & 0.0800 & 0.0390 \\
\hline & & 4 & . & 3,381 & 3.7256 & 3.8685 & 3.9245 & 0.3710 & 0.1566 & 0.0806 & 0.0394 \\
\hline & & 6 & $\dot{.}$ & 3.1209 & 3,5925 & 3.7955 & 3.8864 & 0.4277 & 0.1696 & 0,0838 & 0.0404 \\
\hline Bayes & $\theta$ & $a$ & $\beta$ & \multicolumn{8}{|c|}{ Inverted Gamma distribution $\left(\mathrm{P}_{4}(\mathrm{O} \mid \mathrm{x})\right)$} \\
\hline & 4 & 2 & 0.5 & 3,381 & 3.7256 & 3.8685 & 3.9245 & 0.3710 & 0.1566 & 0.0806 & 0.0394 \\
\hline & & 2 & 1 & 3.4227 & 3,7441 & 3.8781 & 3.9294 & 0.3586 & 0.1541 & 0.0799 & 0.0392 \\
\hline & & 3 & 0.5 & 3.1209 & 3,5925 & 3.7955 & 3.8864 & 0.4277 & 0.1696 & 0.0838 & 0.0404 \\
\hline & & 3 & l & 3.1594 & 3.6104 & 3.805 & 3.8913 & 0.4112 & 0.1660 & 0,0829 & 0,0402 \\
\hline Baves & $\theta$ & $a$ & $\mathrm{~b}$ & \multicolumn{8}{|c|}{ Improper distribution $\left(\mathrm{P}_{\imath}(\theta \mid \mathrm{x})\right)$} \\
\hline & 4 & 1 & l & 3.7338 & 3.8881 & 39542 & 3,9683 & 0.3453 & 0.1517 & 0.0798 & 0.0390 \\
\hline & & l & 2 & 3.8247 & 3.9266 & 3.9738 & 3.9782 & 0.3352 & 0.1499 & 0.0794 & 0.0388 \\
\hline & & l & 3 & 3.9156 & 3.9651 & 3.9934 & 3.9881 & 0.3293 & 0.1488 & 0.0793 & 0.0387 \\
\hline & & 2 & l & 3.4227 & 3,7441 & 3.8781 & 3.9294 & 0.3586 & 0.1541 & 0.0799 & 0,0392 \\
\hline & & 2 & 2 & 3,506 & 3.7812 & 3.8974 & 3.9392 & 0.3362 & 0.1497 & 0.0789 & 0.0389 \\
\hline & & 2 & 3 & 3.5893 & 3.8182 & 3.9166 & 3.949 & 0.3174 & 0.1460 & 0.0780 & 0.0386 \\
\hline & & 3 & l & 3.1594 & 3.6104 & 3.805 & 3.8913 & 0.4112 & 0.1660 & 0.0829 & 0.0402 \\
\hline & & 3 & 2 & 3.2363 & 3.6461 & 3.8238 & 3.901 & 0.3803 & 0.1594 & 0.0811 & 0.0397 \\
\hline & & 3 & 3 & 3,3132 & 3.6818 & 3.8427 & 3.9107 & 0.3524 & 0.1534 & 0.0796 & 0.0392 \\
\hline Bayes & $\theta$ & $\mathrm{c}$ & - & \multicolumn{8}{|c|}{ Non-informative distribution $\left(\mathrm{P}_{k}(\theta \mid x)\right)$} \\
\hline & 4 & 1 & $\dot{-}$ & 4.0072 & 4.0037 & 4.0133 & 3.998 & 0.3964 & 0.1607 & 0.0825 & 0.0395 \\
\hline & & 2 & . & 3.6429 & 3.8497 & 3.9346 & 3.9584 & 0.3594 & 0.1542 & 0.0803 & 0.0391 \\
\hline & & 3 &. & 3,3393 & 3.7071 & 3.8589 & 3.9196 & 3,3393 & 0.1592 & 0.0812 & 0,0396 \\
\hline
\end{tabular}




\section{Under Different Priors \& Two Loss Functions To Compare Bayes Estimators With Some of Classical Estimators For the Parameter of Exponential Distribution}

\subsection{Conclusion}

When we compared the estimated values for $\theta(\theta)$ for the scale parameter of the Exponential distribution by using the methods in this study .We find that Mean Square Errors (MSE) and Mean weighted squared Errors (MWSE) were decreased when sample size increased in all cases. And the MSE increased in all samples sizes (n) when the true value of $\theta$ increased the same thing for MWSE. The best method is the bayes estimation according to the smallest values of MSE for all sample sizes (n) when the prior distribution is

- Inverted Gamma distribution with $(\alpha=3, \beta=1)$, when the true value of $\theta(\theta=0.5)$ see table (4.1).

- Improper distribution with $(\mathbf{a}=3, \mathbf{b}=2)$, when the true value of $\theta(\theta=1)$ see table (4.2).

- Improper distribution with $(a=b=3)$, when the true value of $\theta(\theta=2)$ see table (4.3).

- Improper distribution with $(a=b=3)$, when the true value of $\theta(\theta=4)$ see table (4.4).

The best method is the bayes estimation according to the smallest values of MWSE for all samples sizes (n) when the prior distribution is

- Inverted Gamma distribution with $(\alpha=3, \beta=1), \&$ Improper distribution with $(a=3, b=1)$, when the true value of $\theta(\theta=0.5)$ see table (4.5).

- Improper distribution with $(a=b=3)$, when the true value of $\theta(\theta=1)$ see table (4.6).

- Improper distribution with $(\mathbf{a}=\mathbf{2}, \mathbf{b}=\mathbf{3})$, when the true value of $\theta(\theta=2)$ see table (4.7).

- Gumbel type-II distribution with $b=2 \&$ Improper distribution with ( $a=1$, $\mathbf{b}=3)$, when the true value of $\theta(\theta=4)$ see table (4.8).

\subsection{Recommendations}

we recommend to use the bayes estimation, to estimate scale parameter of the Exponential distribution when the prior distributions are improper distribution and Inverted Gamma distribution and Gumbel type-II distribution with the values of the parameters mentioned in our conclusions. 


\section{Under Different Priors \& Two Loss Functions To Compare Bayes \\ Estimators With Some of Classical Estimators For the \\ Parameter of Exponential Distribution}

\section{References:}

1. Abu-Taleb, A.A., Smadi, M.M. and Alawneh, J.A., (2007), ' Bayes Estimation of the Life time Parameters for the exponential distribution'. Math and Statist, 3(3), 106-108.

2. Al_Kutubi, H.S. (2005), 'On Comparison estimation procedures for parameter and survival function exponential distribution using simulation. $\mathbf{P h}$. D. Thesis, college of Ibn-Al-Hatham, Baghdad University, Iraq.

3. Al_Kutubi, H.S. and Ibrahim, N.A., (2009),' Bayes Estimation for exponential distribution with extension of Jeffery Prior Information', Malaysian Journal of Mathematical Sciences 3(2):297-313.

4. Al_Kutubi, H.S. and Ibrahim, N.A., (2009), 'On the Estimation of Survival Function and Parameter Exponential Life Time Distribution', Journal of Mathematics and Statistics 5 (2):130-135, ISSN 1549-3644.

5. Ali, M.M., Woo, J. and Nadarajah, S. (2005). Bayes estimators of the exponential distribution. J. Statist. Manag. Syst, 8(1), 53-58.

6. Bickel, P.J. \& Doksum, K. A., (1977), " Mathematical Statistics: Basic Ideas and Selected Topics ", Holden- Day, Inc., San Francisco.

7. Elfessi, A. and Reineke, D.M. (2001),' Bayesian look at classical estimation: the exponential distribution', Jour.of stat. Education, 9(1). www. amstat. org/ publications/jse/jse_2001/v9n1/ elfessi.html.

8. Krishnamoorthy, K. (2006), "Handbook of statistical distributions with applications"; Chapman and Hall/ CRC.

9. Ross, M. Sh. (2009),"Introduction to probability and statistics for engineers and scientists ", 3rd Ed. Academic Press.

10. Rossman, A. J., Short, T. H. and Parks, M. T. (1998), "Bayes Estimators for the Continuous Uniform Distribution," Journal of Statistics Education, [Online], 6(3). http://www.amstat.org/publications/jse/v6n3/rossman.html).

11. Tahir, M. and Aslam, M., (2010), ' Bayesian and classical analysis of time to failure model with comparison of uninformative priors '. Pak. J. Statist. Vol. 26(2), 407-415.

12. The Gumbel type-II distribution. (2013). Available at: From Wikipedia, the free encyclopedia. This page was last modified on 10 December 2013, http://en.wikipedia.org/wiki.

13. The inverse gamma distribution. (2015). Available at: From Wikipedia, the free encyclopedia. This page was last modified on 30 October 2015, http://en.wikipedia.org/wiki.

14. The inverse-chi-squared distribution. (2013). Available at: From Wikipedia, the free encyclopedia. This page was last modified on 19 April 2014, http://en.wikipedia.org/wiki.

15. Yang, L. and Zhou, H. and Yuan, S. (2013), ' Bayes Estimation of Parameter of Exponential Distribution under a Bounded Loss Function'. Research Journal of Mathematics and Statistics 5(4): 28-31, ISSN: 2042-2024, e-ISSN: 2040-7505. 


\section{Under Different Priors \&Two Loss Functions To Compare Bayes Estimators With Some of Classical Estimators For the \\ Parameter of Exponential Distribution}

\section{Appendix-A: The posterior distribution using different Priors}

1. The posterior distribution using Letr distribution as prior.

It is asermed that $\theta$ follows the Lavy distribution with pdf as given below:

$\mathrm{P}(\theta) a \sqrt{\frac{b_{2}}{2 \pi}} \theta^{\frac{3}{2}}$ exp $\left(-\frac{b_{2}}{2 \theta}\right)$ for $b_{y}, \theta>0$

Where $b_{3}=$ hypeparametes

Then the posterior distribution of given the data $\underline{x}=\left(x_{1}, x_{2}, \ldots, x_{n}\right)$ is:

$$
P(\theta \backslash x)=\frac{L(\underline{x} \backslash \theta) P(\theta)}{\int L(\underline{x} \backslash \theta) P(\theta) d \theta}
$$

Substituting the equation (2) and the equation (A.1) in equation (A.2), we get:

$$
\mathrm{P}_{1}(\theta \backslash \mathrm{x})=\frac{\theta^{-\mathrm{n}} \exp \left(-\frac{\sum_{\mathrm{i}=1}^{\mathrm{n}} \mathrm{x}_{\mathrm{i}}}{\theta}\right)\left[\sqrt{\frac{b_{3}}{2 \pi}} \theta^{-\frac{3}{2}} \exp \left(-\frac{b_{3}}{2 \theta}\right)\right]}{\int_{0}^{\infty} \theta^{-\mathrm{n}} \exp \left(-\frac{\sum_{\mathrm{i}=1}^{\mathrm{n}} \mathrm{x}_{\mathrm{i}}}{\theta}\right)\left[\sqrt{\frac{b_{3}}{2 \pi}} \theta^{-\frac{3}{2}} \exp \left(-\frac{b_{3}}{2 \theta}\right)\right] d \theta}
$$

$P_{i}(\theta \backslash x)=\frac{\theta^{-n-\frac{3}{2}} \exp \left(-\frac{1}{\theta}\left(\sum_{i=1}^{n} x_{i}+\frac{b_{i}}{2}\right)\right)}{\int_{0}^{\infty} \theta^{-n-\frac{3}{2}} \exp \left(-\frac{1}{\theta}\left(\sum_{i=1}^{n} x_{i}+\frac{b_{i}}{2}\right)\right) d \theta}$

We can write $\theta^{-a+\frac{3}{2}} a \theta^{-\left[\left(a+\frac{1}{2}\right)+1\right]}$, and by multiplying the integral in equation (A.4) by the quantity which equals to

$$
\left(\frac{\left(\sum_{i=1}^{n} x_{i}+\frac{b_{3}}{2}\right)^{\left(n+\frac{1}{2}\right)}}{\Gamma\left(n+\frac{1}{2}\right)}\right)\left(\frac{\Gamma\left(n+\frac{1}{2}\right)}{\left(\sum_{i=1}^{n} x_{i}+\frac{b_{3}}{2}\right)^{\left(n+\frac{1}{2}\right)}}\right) \text {, where } \Gamma(.) \text { is a gamma function }
$$

Then we get,

$$
P_{1}(\theta \backslash x)=\frac{\left(\sum_{i=1}^{n} x_{i}+\frac{b_{j}}{2}\right)^{\left(a+\frac{1}{2}\right)} \theta^{-\left[\left(a+\frac{1}{2}\right)+1\right]} \exp \left(-\frac{1}{\theta}\left(\Sigma_{i=1}^{A} x_{i}+\frac{b_{3}}{2}\right)\right)}{\Gamma\left(n+\frac{1}{2}\right) A(x ; \theta)}
$$

Where $A(x \oplus)$ equals to
$A(x ; \theta)-\int_{0}^{\infty} \frac{\left(\sum_{i=1}^{n} x_{i}+\frac{b_{i}}{2}\right)^{\left(a+\frac{1}{2}\right)} \theta^{-\left[\left(a+\frac{1}{2}\right)+1\right]} \exp \left(-\frac{1}{\theta}\left(\sum_{i}^{A}-1 x_{i}+\frac{b_{3}}{2}\right)\right)}{\Gamma\left(n+\frac{1}{2}\right)} d \theta-1$. Be the integral of the pdf of the Inverted Gamma distribution. Then we get the posterior distribution of $\theta$ given the dats $\underline{x}=\left(x_{1}, x_{2}, \ldots, x_{n}\right)$ is

$$
\begin{aligned}
& P_{1}(\theta \backslash x)=\frac{\left(\sum_{i=1}^{n} x_{i}+\frac{b_{z}}{2}\right)^{\left(n+\frac{1}{2}\right)}}{\Gamma\left(n+\frac{1}{2}\right)} \theta^{\left[\left(n+\frac{1}{2}\right)+1\right]} \exp \left(-\frac{1}{\theta}\left(\sum_{i=1}^{n} x_{i}+\frac{b_{z}}{2}\right)\right) \\
& \text { It means that } P(\theta \backslash \mathbf{x}) \sim \text { Inverted Gamma distribution with new } \\
& \text { parameters }\left(a_{n=00}=\left(n+\frac{1}{2}\right), \beta_{n=n s}=\left(\sum_{i=1}^{n} x_{1}+\frac{b_{i}}{2}\right)\right) \text {. }
\end{aligned}
$$

2. The posterior distribution using Gumbel tope-II distribution as prior:

It is assumed that $\theta$ follows the Gumbel type-I distribution with pdf as given below: $P(\theta) a$ a $b \theta^{-(z+1)} \exp \left(-\frac{b}{\theta^{2}}\right)$ for $a, b, \theta>0$

If $a=1$ then we get

$P(\theta) a \quad b \theta^{-2} \quad \exp \left(-\frac{b}{\theta}\right)$ for $b, \theta>0$

Then the posterior distribution of given the data $\underline{x}=\left(x_{1}, x_{2}, \ldots, x_{n}\right)$ according to the equation (A.2), we get it by substituting the equation (2) and the equation (A.8) in equation (A.2), so we have

$$
\begin{aligned}
& \mathrm{P}_{2}(\theta \backslash \mathrm{x})=\frac{\theta^{-\mathrm{n}} \exp \left(-\frac{\sum_{\mathrm{i}=1}^{\mathrm{n}} \mathrm{x}_{\mathrm{i}}}{\theta}\right)\left[\mathrm{b} \theta^{-2} \exp \left(-\frac{\mathrm{b}}{\theta}\right)\right]}{\int_{0}^{\infty} \theta^{-\mathrm{n}} \exp \left(-\frac{\sum_{\mathrm{i}-1}^{\mathrm{n}} \mathrm{x}_{\mathrm{i}}}{\theta}\left[\mathrm{b} \theta^{-2} \exp \left(-\frac{\mathrm{b}}{\theta}\right)\right] d \theta\right.} \\
& \mathrm{P}_{2}(\theta \backslash \mathrm{x})=\frac{\theta^{-\mathrm{n}-2} \exp \left(-\frac{1}{\theta}\left(\sum_{\mathrm{i}-1}^{\mathrm{n}} \mathrm{x}_{\mathrm{i}}+\mathrm{b}\right)\right)}{\int_{0}^{-\mathrm{n}-2} \exp \left(-\frac{1}{\theta}\left(\sum_{\mathrm{i}-1}^{\mathrm{n}} \mathrm{x}_{\mathrm{i}}+\mathrm{b}\right)\right) d \theta}
\end{aligned}
$$

We can write $\theta^{-n-2}$ as $\theta^{-[(a+1)+1]}$, and by multiplying the integral in equation (A.10) by the quantity which equals to

$\left(\frac{\left(\sum_{i=1}^{n} x_{i}+b\right)^{(n+1)}}{\Gamma(n+1)}\right)\left(\frac{\Gamma(n+1)}{\left(\sum_{i=1}^{n} x_{i}+b\right)^{(n+1)}}\right)$, where $\Gamma($.$) is a gamma function . Then we$

$P_{2}(\theta \backslash x)-\frac{\left(\sum_{i=1}^{n} x_{i}+b\right)^{(n+1)} \theta^{-[(n+1)+1]} \exp \left(\cdot \frac{1}{\theta}\left(\sum_{i=1}^{n} x_{i}+b\right)\right)}{\Gamma(n+1) B(x ; \theta)}$ 


\section{Under Different Priors \&Two Loss Functions To Compare Bayes Estimators With Some of Classical Estimators For the \\ Parameter of Exponential Distribution}

Where $B(x, t)$ equals to

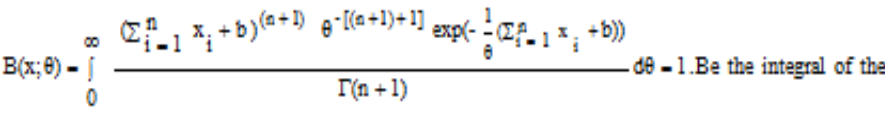
pdf of the Inverted Gamma distribution. Then we get the posterior distribution of $\theta$ given the data $\underline{x}=\left(x_{1}, x_{2}, \ldots, x_{n}\right)$ is

$P_{2}(\theta \backslash x)=\frac{\left(\sum_{i=1}^{n} x_{i}+b\right)^{(n+1)} \theta^{-[(n+1)+1]} \exp \left(-\frac{1}{\theta}\left(\sum_{i=1}^{\mathrm{a}}=1 x_{i}+b\right)\right)}{\Gamma(n+1)}$

It means that $P_{2}(\theta \mid x) \sim$ Inverted Gamma distribution with new parameters $\left(\alpha_{\text {man }}=(n+1), \beta_{(n=w)}-\left(\sum_{i=1}^{n} x_{1}+b\right)\right)$.

3. The posterior distribution using Inverse Chi-square distribution as prior: It is asumed that $\theta$ follows the Inverse Chi-square distribution with pdf as given below:

$\mathrm{P}(\theta) \propto \frac{1}{2^{\frac{y}{2}}} \theta^{-\frac{y}{2}-1} \exp \left(-\frac{1}{2 \theta}\right) \quad$ for $\quad \mathrm{v}, \theta>0$

Then the posterior distribution of given the data $\underline{x}=\left(x_{1}, x_{2}, \ldots, x_{n}\right)$ according to the equation (A.2), we get it by substituting the equation (2) and the equation (A.13) in equation (A.2), 80 we have

$\mathrm{P}_{2}(\theta \backslash \mathrm{x})=\frac{\theta^{-\mathrm{n}} \exp \left(-\frac{\sum_{\mathrm{i}-1}^{\mathrm{n}} \mathrm{x}_{\mathrm{i}}}{\theta}\right)\left[\frac{1}{2^{\frac{\gamma}{2}}} \theta^{-\frac{\mathrm{v}}{2}-1} \exp \left(-\frac{1}{2 \theta}\right)\right]}{\int_{0}^{\infty} \theta^{-\mathrm{n}} \exp \left(-\frac{\sum_{\mathrm{i}-1}^{\mathrm{n}} \mathrm{x}_{\mathrm{i}}}{\theta}\right)\left[\frac{1}{2^{\frac{\gamma}{2}}} \theta^{-\frac{\mathrm{v}}{2}-1} \exp \left(-\frac{1}{2 \theta}\right)\right] d \theta}$

$P_{i}(\theta \backslash x)=\frac{\theta^{-\left[\left(n+\frac{v}{2}\right)+1\right]} e^{-\frac{1}{\theta}\left(\sum_{i=1}^{n} x_{i}+\frac{1}{2}\right)}}{\int_{0}^{\infty} e^{-\left[\left(n+\frac{v}{2}\right)+1\right]} e^{-\frac{1}{\theta}\left(\sum_{i=1}^{n} x_{i}+\frac{1}{2}\right)} d \theta}$

By multiplying the integral in equation (A.15) by the quantity which equals to

$\left(\frac{\left(\sum_{i-1}^{n} x_{i}+\frac{1}{2}\right)^{\left(n-\frac{3}{2}\right)}}{\Gamma\left(n+\frac{v}{2}\right)}\right)\left(\frac{\Gamma\left(n+\frac{v}{2}\right)}{\left(\sum_{i-1}^{n} x_{i}+\frac{1}{2}\right)^{\left(n+\frac{3}{2}\right)}}\right)$, where $\Gamma($.$) is a gamma function. Then we$

$P_{1}(\theta \backslash x)=\frac{\left(\sum_{i=1}^{n} x_{i}+\frac{1}{2}\right)^{\left(n+\frac{3}{2}\right)} \theta^{-\left[\left(n-\frac{v}{2}\right)+1\right]} \exp \left(-\frac{1}{\theta}\left(\sum_{i=1}^{n} x_{i}+\frac{1}{2}\right)\right)}{\Gamma\left(n+\frac{v}{2}\right) C(x ; \theta)}$
Where $C(x ; \theta)$ equals to

$C(x ; \theta)=\int_{0}^{\infty} \frac{\left(\sum_{i=1}^{n} x_{i}+\frac{1}{2}\right)^{\left(n+\frac{v}{2}\right)} \theta^{-\left[\left(n+\frac{v}{2}\right)+1\right]} \exp \left(-\frac{1}{\theta}\left(\sum_{i=1}^{n} x_{i}+\frac{1}{2}\right)\right)}{\Gamma\left(n+\frac{v}{2}\right)} d \theta-1$. Be the integral of the pdf of the Inverted Gemma distribution. Then we get the posterior distribution of $\theta$ given the data $\underline{x}=\left(x_{1}, x_{2}, \ldots, x_{n}\right)$ is

$P_{i}(\theta \backslash \mathrm{x})=\frac{\left(\Sigma_{i=1}^{\mathrm{n}} \mathrm{x}_{\mathrm{i}}+\frac{1}{2}\right)^{\left(a+\frac{V}{2}\right)}}{\Gamma\left(\mathrm{n}+\frac{\mathrm{v}}{2}\right)} \theta^{\left[\left(\mathrm{a}+\frac{\mathrm{V}}{2}\right)+1\right]} \exp \left(-\frac{1}{\theta}\left(\sum_{i=1}^{\mathrm{n}} \mathrm{x}_{1}+\frac{1}{2}\right)\right)$

It means that $\left.\mathrm{P}_{j}(\theta \backslash \mathrm{x})\right)$ ) Inverted Gemma distribution with new parameters $\left(\alpha_{\text {(nww) }}-\left(\mathrm{n}+\frac{v}{2}\right), \beta_{(\mathrm{ww})}-\left(\sum_{i=1}^{\mathrm{n}} \mathrm{x}_{1}+\frac{1}{2}\right)\right)$.

4. Theposterior distribution using Inverted Gamma distribution as prior:

It is assumed that $\theta$ follows the Inverted Gamma distribution with pdf as given below:

$P(\theta) \alpha \frac{\beta^{\alpha}}{\Gamma \alpha} \theta^{-(\alpha+1)} \exp \left(-\frac{\beta}{\theta}\right) \quad$ for $\alpha, \beta, \theta>0$

Then the posterior distribution of given the data $\underline{x}=\left(\mathrm{x}_{1}, \mathrm{x}_{2}, \ldots, \mathrm{x}_{\mathrm{a}}\right)$ according to the equation (A.2), we get it by substituting the equation (2) and the equation (A.18) in equation (A.2), 30 we have

$\mathrm{P}_{s}(\theta \backslash \mathrm{x})=\frac{\theta^{-\mathrm{n}} \exp \left(-\frac{\sum_{\mathrm{i}-1}^{\mathrm{n}} \mathrm{x}_{\mathrm{i}}}{\theta}\right)\left[\frac{\beta^{\alpha}}{\Gamma \alpha} \theta^{-(\alpha+1)} \exp \left(-\frac{\beta}{\theta}\right)\right]}{\int_{0}^{\infty} \theta^{-\mathrm{n}} \exp \left(-\frac{\sum_{\mathrm{i}=1}^{\mathrm{n}} \mathrm{x}_{\mathrm{i}}}{\theta}\right)\left[\frac{\beta^{\alpha}}{\Gamma \alpha} \theta^{-(\alpha+1)} \exp \left(-\frac{\beta}{\theta}\right)\right] d \theta}$

$P_{4}(\theta \backslash x)=\frac{\theta^{-[(n+\alpha)+1]} \exp \left(-\frac{1}{\theta}\left(\sum_{i=1}^{n} x_{i}+\beta\right)\right)}{\int_{0}^{\infty} \theta^{-[(n+\alpha)+1]} \exp \left(-\frac{1}{\theta}\left(\sum_{i=1}^{n} x_{i}+\beta\right) d \theta\right.}$

By multiplying the integral in equation (A.20) by the quantity which equals to

$\left(\frac{\left(\sum_{\mathrm{i}=1}^{\mathrm{n}} \mathrm{x}_{\mathrm{i}}+\beta\right)^{(n+2)}}{\Gamma(\mathrm{n}+\alpha)}\right)\left(\frac{\Gamma(\mathrm{n}+\alpha)}{\left(\sum_{\mathrm{i}=1}^{\mathrm{n}} \mathrm{x}_{\mathrm{i}}+\beta\right)^{(n+2)}}\right)$, where $\Gamma($.$) is a gamma function. Then we get,$

$P_{s}(\theta \backslash x)=\frac{\left(\sum_{i=1}^{n} x_{i}+\beta\right)^{(n+\alpha)} \theta^{-[(n+\alpha)+1]} \exp \left(-\frac{1}{\theta}\left(\sum_{i=1}^{n} x_{i}+\beta\right)\right.}{\Gamma(n+\alpha) D(x ; \theta)}$

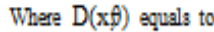




\section{Under Different Priors \&Two Loss Functions To Compare Bayes Estimators With Some of Classical Estimators For the Parameter of Exponential Distribution}

$D(x ; \theta)=\int_{0}^{\infty} \frac{\left(\sum_{i=1}^{n} x_{i}+\beta\right)^{(n+\alpha)} \theta^{-[(n+\alpha)+1]} \exp \left(-\frac{1}{\theta}\left(\sum_{i=1}^{n} x_{i}+\beta\right)\right)}{\Gamma(n+\alpha)} d \theta-1$. Be the integral of the pdf of the Inverted Gamma distribution. Then we get the posterior distribution of $\theta$ given the data $\underline{x}=\left(x_{1}, x_{2}, \ldots, x_{n}\right)$ is

$P_{s}(\theta \backslash x)=\frac{\left(\sum_{i=1}^{n} x_{i}+\beta\right)^{(n+\alpha)} \theta^{-[(n+\alpha)+1]} \exp \left(-\frac{1}{\theta}\left(\sum_{i=1}^{n} x_{i}+\beta\right)\right)}{\Gamma(n+\alpha)}$

It means that $P_{6}(\theta \mid \mathbf{x})$ Inverted Gamma distribution with new

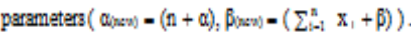

5. The posterior distribution using improper distribution as prior:

It is asumed that $\theta$ follows the improper distribution with pdf as given below:

$P(\theta) a \cdot \theta^{-(a+1)} \exp \left(-\frac{b}{\theta}\right) \quad$ for $b, \theta>0$ and $-\infty<a<\infty$

Then the posterior distribution of given the data $\underline{x}=\left(x_{1}, x_{2}, \ldots, x_{n}\right)$ according to the equation (A.2), we get it by substituting the equation (2) and the equation (A.22) in equation (A.2), 30 we have

$$
\begin{aligned}
& \mathrm{P}_{s}(\theta \backslash \mathrm{x})=\frac{\theta^{-\mathrm{n}} \exp \left(-\frac{\sum_{\mathrm{i}=1}^{\mathrm{n}} \mathrm{x}_{\mathrm{i}}}{\theta}\right)\left[\theta^{-(\mathrm{a}+1)} \exp \left(-\frac{b}{\theta}\right)\right]}{\int_{0}^{\infty} \theta^{-\mathrm{n}} \exp \left(-\frac{\sum_{\mathrm{i}=1}^{\mathrm{n}} \mathrm{x}_{\mathrm{i}}}{\theta}\right)\left[\theta^{-(\mathrm{a}+1)} \exp \left(-\frac{b}{\theta}\right)\right] d \theta} \\
& P_{s}(\theta \backslash x)=\frac{\theta^{-[(n+a)+1]} \exp \left(-\frac{1}{\theta}\left(\sum_{i=1}^{n} x_{i}+b\right)\right)}{\int_{0}^{\infty} \theta^{-[(n+a)+1]} \exp \left(-\frac{1}{\theta}\left(\sum_{i=1}^{n} x_{i}+b\right)\right) d \theta}
\end{aligned}
$$

By multiplying the integral in equation (A.25) by the quantity which equals to

$$
\left(\frac{\left(\sum_{i=1}^{n} x_{i}+b\right)^{(n+2)}}{\Gamma(n+a)}\right)\left(\frac{\Gamma(n+a)}{\left(\sum_{i=1}^{n} x_{i}+b\right)^{(n+2)}}\right) \text {, where } \Gamma(.) \text { is a gamma function. Then we get, }
$$$$
P_{s}(\theta \backslash x)=\frac{\left(\sum_{i=1}^{n} x_{i}+b\right)^{(n+2)} \theta^{-[(n+a)+1]} \exp \left(-\frac{1}{\theta}\left(\sum_{i=1}^{n} x_{i}+b\right)\right)}{\Gamma(n+a) E(x ; \theta)}
$$

Where $E(x \phi)$ equals to

$E(x ; \theta)-\int_{0}^{\infty} \frac{\left(\sum_{i=1}^{n} x_{i}+b\right)^{(n+a)} \theta^{-[(n+a)+1]} \exp \left(-\frac{1}{\theta}\left(\sum_{i=1}^{n} x_{i}+b\right)\right)}{\Gamma(n+a)} d \theta-1$. Be the integral of the pdf of the Inverted Gamma distribution. Then we get the posterior distribution of $\theta$ given the data $\underline{x}=\left(x_{1}, x_{2}, \ldots, x_{n}\right)$ is
$P_{s}(\theta \backslash x)=\frac{\left(\sum_{i=1}^{n} x_{i}+b\right)^{(n+a)} \theta^{-[(n+a)+1]} \exp \left(-\frac{1}{\theta}\left(\sum_{i=1}^{n} x_{i}+b\right)\right)}{\Gamma(n+a)}$

It means that $P_{s}(\theta \backslash x) \sim$ Inverted Gemma distribution with new parameters $\left(\alpha_{\text {new })}-(n+a), \beta(n=w)=\left(\sum_{i-1}^{n} x_{1}+b\right)\right)$.

6. Theposterior distribution using Non-informative distribution as prior

It is assumed that $\theta$ follows the non-informative distribution with pdf as given below:

$\mathrm{P}(\theta)$ a $\frac{1}{\theta^{c}} \quad$ for $\theta, c>0$

Then the posterior distribution of given the data $\underline{x}=\left(x_{1}, x_{2}, \ldots, x_{n}\right)$ according to the equation (A.2), we get it by substituting the equation (2) and the equation (A.28) in equation (A.2), 80 we have

$\mathrm{P}_{6}(\theta \backslash \mathrm{x})=\frac{\theta^{-\mathrm{n}} \exp \left(-\frac{\sum_{\mathrm{i}=1}^{\mathrm{n}} \mathrm{x}_{\mathrm{i}}}{\theta}\right)\left[\theta^{-c}\right]}{\int_{0}^{\infty} \theta^{-\mathrm{n}} \exp \left(-\frac{\sum_{\mathrm{i}=1}^{\mathrm{n}} \mathrm{x}_{\mathrm{i}}}{\theta}\right)\left[\theta^{-c}\right] d \theta}$
$\mathrm{P}_{6}(\theta \backslash \mathrm{x})=\frac{\theta^{-(\mathrm{n}+c)} \exp \left(-\frac{1}{\theta} \sum_{\mathrm{i}=1}^{\mathrm{n}} \mathrm{x}_{\mathrm{i}}\right)}{\int_{0}^{\infty} \theta^{-(\mathrm{n}+c)} \exp \left(-\frac{1}{\theta} \sum_{\mathrm{i}=1}^{\mathrm{n}} \mathrm{x}_{\mathrm{i}}\right) d \theta}$

We can write $\theta^{-(a+c)}$ as $\theta^{-[(a+c-1)+1]}$, and by multiplying the integral in equation (A.30), by the quantity which equals to

$\left(\frac{\left(\sum_{\mathrm{i}-1}^{\mathrm{n}} \mathrm{x}_{\mathrm{i}}\right)^{(\mathrm{n}+\mathrm{c}-\mathrm{l})}}{\Gamma(\mathrm{n}+\mathrm{c}-1)}\right)\left(\frac{\Gamma(\mathrm{n}+\mathrm{c}-1)}{\left(\sum_{\mathrm{i}-1}^{\mathrm{n}} \mathrm{x}_{\mathrm{i}}\right)^{(\mathrm{n}+\infty-1)}}\right)$, where $\Gamma($.$) is a gamma function. Then we get$

$\mathrm{P}_{f}(\theta \backslash \mathrm{x})=\frac{\left(\sum_{\mathrm{i}-1}^{\mathrm{n}} \mathrm{x}_{\mathrm{i}}\right)^{(n+-1)} \theta^{-[(\mathrm{n}+c-1)+1]} \exp \left(-\frac{1}{\theta} \sum_{\mathrm{i}-1}^{\mathrm{n}} \mathrm{x}_{\mathrm{i}}\right)}{\Gamma(\mathrm{n}+\mathrm{c}-1) \mathrm{F}(\mathrm{x} ; \theta)}$

Where $F(x, \rho)$ equals to

$F(x ; \theta)=\int_{0}^{\infty} \frac{\left(\sum_{i=1}^{n} x_{i}\right)^{(n+c-1)} \theta^{-[(n+c-1)+1]} \exp \left(-\frac{1}{\theta} \sum_{i=1}^{n} x_{i}\right)}{\Gamma(n+c-1)} d \theta-1$. Be the integral of the pdf of the Inverted Gemma distribution. Then we get the posterior distribution of $\theta$ given the data $\underline{x}=\left(x_{1}, x_{2}, \ldots, x_{n}\right)$ is

$P_{6}(\theta \backslash x)=\frac{\left(\sum_{i=1}^{n} x_{i}\right)^{(n+c-1)} \theta^{-[(n+c-1)+1]} \exp \left(-\frac{1}{\theta} \sum_{i=1}^{n} x_{i}\right)}{\Gamma(n+c-1)}$

It means that $P_{6}(\theta \mid x) \sim$ Inverted Gamma distribution with new parameters $\left.\left(\alpha_{j m w}\right)=(n+c-1), \beta(n=w)=\left(\sum_{i=1}^{n} x_{i}\right)\right)$. 


\section{Under Different Priors \&Two Loss Functions To Compare Bayes Estimators With Some of Classical Estimators For the Parameter of Exponential Distribution}

Appendix-B: The following is the derivation of these estimators under the squared error loss function.

1. Thesquared error loss function

To obtain the Bayes' estimator, we minimize the posterior expected loss given by:

$L_{1}(\theta, \theta)-(\theta-\theta)^{2}$, the riak function is:

$R(\theta-\theta)-E\left[L_{1}(\theta, \theta)\right]$

$R(\theta-\theta)-\int L_{1}(\theta, \theta) P(\theta \backslash x) d \theta$

$$
\theta
$$

$R(\theta-\theta)-\int(\theta-\theta)^{2} \mathrm{P}(\theta \backslash \mathrm{x}) d \theta \Rightarrow \mathrm{R}(\theta-\theta)-\left[\left(\theta^{2}-2 \theta \hat{\theta}+\theta^{2}\right) \mathrm{P}(\theta \backslash \mathrm{x}) d \theta\right.$

$\theta \quad \theta$

$R(\theta-\theta)=\theta^{2}|P(\theta \backslash x) d \theta-2 \theta| \theta P(\theta \mid x) d \theta+\mid \theta^{2} P(\theta \mid x) d \theta \Rightarrow$

$R(\theta-\theta)-\theta^{2}-2 \theta E(\theta \mid x)+B\left(\theta^{2} \backslash x\right)$

Lat $\frac{\partial}{\wedge} R(\theta-\theta)-0$, we get Bayes estimator of $\theta$ denoted by $\hat{\theta}_{\text {ayje }}$ for the above prior as $\partial \theta$

follows

$\hat{\theta}_{\mathrm{gz}}-\mathrm{E}(\theta \backslash \mathrm{x})-\int_{0}^{\tilde{E}} \theta \mathrm{P}(\theta \backslash \mathrm{x}) \mathrm{d \theta}$

1.1 Baves estimation using Lerr distribution as prior

To obtain the Bayes' estimator under Levy distribution as prior. Substituting the equation (A.6) in equation (B.3), we get:

$\hat{\theta}_{m: 1}=\int_{0}^{e} \theta P_{1}(\theta \backslash x) d \theta$

$\hat{\theta}_{m=1}-\int_{0}^{e} \theta \frac{\left(\sum_{i=1}^{n} x_{i}+\frac{b_{i}}{2}\right)^{\left(n+\frac{1}{2}\right)}}{\Gamma\left(n+\frac{1}{2}\right)} \theta^{-\left[\left(n+\frac{1}{2}\right)+1\right]} \exp \left(-\frac{1}{\theta}\left(\sum_{i=1}^{n} x_{1}+\frac{b_{2}}{2}\right)\right) d \theta$

$\hat{\theta}_{m=1}=\int_{0}^{\infty} \frac{\left(\sum_{i=1}^{n} x_{i}+\frac{b_{2}}{2}\right)^{\left(n+\frac{1}{2}\right)}}{\Gamma\left(n+\frac{1}{2}\right)} \theta^{-\left[\left(n+\frac{1}{2}\right)+1\right]+1} \exp \left(-\frac{1}{\theta}\left(\sum_{i=1}^{n} x_{1}+\frac{b_{2}}{2}\right)\right) d \theta$

For the equation (45), we can write- $\left[\left(n+\frac{1}{2}\right)+1\right]+1=-\left[n+\frac{1}{2}+1-1\right]--\left[\left(n-\frac{1}{2}\right)+1\right]$ That is $\left(\mathrm{n}+\frac{1}{2}\right)=\mathrm{n}+\frac{1}{2}+\frac{1}{2}-\frac{1}{2}-\left[\left(\mathrm{n}-\frac{1}{2}\right)+1\right]$.

By multiplying the integral in equation (B.5) by the quantity which equals to $\mathrm{Al}-\left(\frac{\Gamma\left(\mathrm{n}-\frac{1}{2}\right)}{\Gamma\left(\mathrm{n}-\frac{1}{2}\right)}\right)$

where $\Gamma($.$) is a gamma function.$
Then, we have

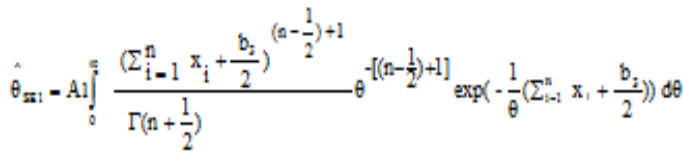

Then, we have

$\hat{\theta}_{\mathrm{min} 1}=\frac{\Gamma\left(\mathrm{n}-\frac{1}{2}\right)}{\Gamma\left(\mathrm{n}+\frac{1}{2}\right)}\left(\sum_{i=1}^{\mathrm{n}} \mathrm{x}_{i}+\frac{\mathrm{b}_{i}}{2}\right)(\mathrm{Ar}(\mathrm{x} ; \theta))$

Where $A 2(x, f)$ equals to

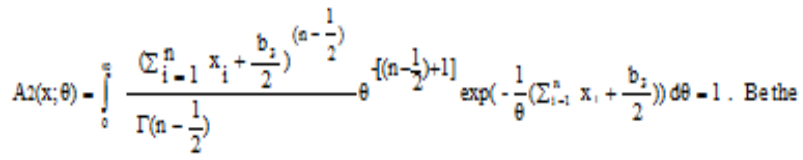

integral of the pdf of the Inverted Gemma distribution. Then we get the Bayes estimator of $\theta$ as the following formula:

$\hat{\theta}_{\mathrm{ma1}}=\frac{\Gamma\left(\mathrm{n}-\frac{1}{2}\right)}{\Gamma\left(\mathrm{n}+\frac{1}{2}\right)}\left(\sum_{i=1}^{\mathrm{n}} \mathrm{x}_{1}+\frac{\mathrm{b}_{j}}{2}\right) \quad, \mathrm{n} \& \mathrm{~b}_{2}>0$

1.2 Baves estimation using Gumbel tope-II distribution as prior.

To obtain the Bayes' estimator under the Gumbel type-I distribution as prior distribution. Substituting the equation (A.12) in equation (B.3), we get:

$\hat{\theta}_{m z:}=\int_{0}^{e} \theta P_{2}(\theta \backslash x) d \theta$

$\hat{\theta}_{m=2}=\int_{0}^{\int} \theta \frac{\left(\sum_{i=1}^{n} x_{i}+b\right)^{(n+1)} \theta^{-[(n+1)+1]} \exp \left(\cdot \frac{1}{\theta}\left(\sum_{i=1}^{n} x_{i}+b\right)\right)}{\Gamma(n+1)} d \theta$

$\hat{\theta}_{m 2}=\int_{0}^{\int} \frac{\left(\sum_{i=1}^{n} x_{i}+b\right)^{(n+1)} \theta^{-[(n+1)+1-1]} \exp \left(\cdot \frac{1}{\theta}\left(\Sigma_{i=1}^{a} x_{i}+b\right)\right)}{\Gamma(n+1)} d \theta$

For the equation $(B \cdot 10)$, we can write $-[(n+1)+1-1]=-[n+1]$ And by multiplying the integral in equation (B.10) by the quantity which equals to $\mathrm{Bl}=\left(\frac{\Gamma(\mathrm{n})}{\Gamma(\mathrm{n})}\right)$, where $\Gamma($.$) is a gamma function.$ Then, we have

$\hat{\theta}_{m=2}-B i \int_{0}^{\infty} \frac{\left(\sum_{i=1}^{n} x_{i}+b\right)^{(n+1)} \theta^{-(n+1)} \exp \left(\cdot \frac{1}{\theta}\left(\sum_{i=1}^{A} x_{i}+b\right)\right)}{\Gamma(n+1)} d \theta$

Then we have

$\hat{\theta}_{m 2}=\frac{\Gamma(n)}{\Gamma(n+1)}\left(\sum_{i=1}^{n} x_{1}+b\right)(B 2(x ; \theta))$

Where $\mathrm{B} 2(\mathrm{x}, \hat{f})$ equals to 


\section{Under Different Priors \& Two Loss Functions To Compare Bayes Estimators With Some of Classical Estimators For the Parameter of Exponential Distribution}

$\operatorname{Bl}(x ; \theta)-\int_{0}^{0} \frac{\left(\sum_{\mathrm{i}=1}^{\mathrm{n}} x_{\mathrm{i}}+b\right)^{2} \theta^{-(a+1)} \exp \left(\cdot \frac{1}{\theta}\left(\sum_{\mathrm{i}}^{\mathrm{a}}-1 x_{\mathrm{i}}+b\right)\right)}{\Gamma(\mathrm{n})} d \theta-1$. Be the integral of the pdf of the Inverted Gamma distribution. Then we get the Bayes estimator of $\theta$ as the following formula: $\hat{\theta}_{m: 2}=\frac{\Gamma(n)}{\Gamma(n+1)}\left(\Gamma_{i=1}^{n} x_{i}+b\right) \quad, n \& b>0$

1.3 Baves estimation using Inverse chi-squared distribution as prior.

To obtain the Bayes' estimator under inverse chi-squared distribution as prior. Substituting the equation (A.16) in equation (B.3), we get:

$\hat{\theta}_{m i}=\int_{0}^{e} \theta P_{i}(\theta \mid x) d \theta$

$\hat{\theta}_{m=2}=\int_{0}^{\int} \theta \frac{\left(\sum_{i=1}^{n} x_{i}+\frac{1}{2}\right)^{\left(n+\frac{y}{2}\right)}}{\Gamma\left(n+\frac{\eta}{2}\right)} \theta^{\left[\left(n+\frac{y}{2}\right)+1\right]} \exp \left(-\frac{1}{\theta}\left(\sum_{i=1}^{n} x_{1}+\frac{1}{2}\right)\right) d \theta$

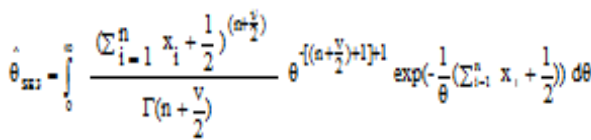

For the equation $(\mathrm{B} .15)$, we can write $-\left[\left(\left(\mathrm{n}+\frac{\mathrm{v}}{2}\right)+1\right)-1\right]--\left[\left(\mathrm{n}+\frac{\mathrm{v}}{2}-1\right)+1\right]$ And by multiplying the integral in equation (B.15) by the quantity which equals to $\mathrm{Cl}-\left(\frac{\Gamma\left(\mathrm{n}+\frac{\mathrm{v}}{2}-1\right)}{\Gamma\left(\mathrm{n}+\frac{\mathrm{v}}{2}-1\right)}\right)$, where $\Gamma($.$) is a$ gamma function

Then, we have

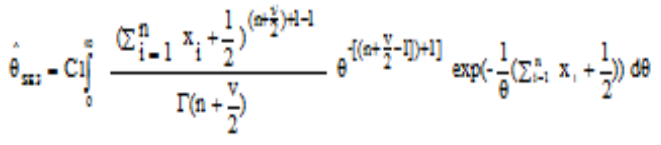

Then we have

$\hat{\theta}_{m=1}=\frac{\Gamma\left(\mathrm{n}+\frac{v}{2}-1\right)}{\Gamma\left(\mathrm{n}+\frac{\eta}{2}\right)}\left(\sum_{i=1}^{\mathrm{n}} \mathrm{x}_{1}+\frac{1}{2}\right)(\mathrm{C} 2(\mathrm{x} ; \theta))$

Where $C_{2}(x, 0)$ equals to

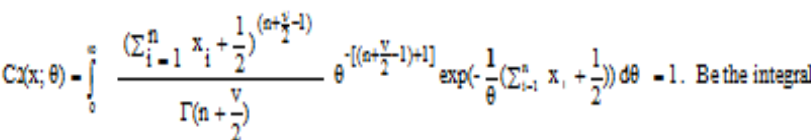

of the pdf of the Inverted Gramma distribution. Then we gat the Bayes estimator of $\theta$ as the following formula $\hat{\theta}_{m i}=\frac{\Gamma\left(n+\frac{v}{2}-1\right)}{\Gamma\left(n+\frac{v}{2}\right)}\left(\sum_{i 2}^{n} x_{1}+\frac{1}{2}\right), n \& v>0$

1.4 Bars estimation using Inverted gamma distribution as prior.

To obtain the Baysa' estimator under the inverted gamma distribution as prior. Substituting the equation (A.21) in equation (B.3), we get:

$\hat{\theta}_{\mathrm{sin}}=\int_{0}^{e} \theta \mathrm{P}_{4}(\theta \backslash \mathrm{x}) \mathrm{d \theta}$

$\hat{\theta}_{m=1}=\int_{0}^{\theta} \theta \frac{\left(\sum_{i=1}^{n} x_{i}+\beta\right)^{(n+\alpha)} \theta^{-[(n+\alpha)+1]} \exp \left(-\frac{1}{\theta}\left(\Sigma_{i=1}^{n} x_{i}+\beta\right)\right)}{\Gamma(n+\alpha)} d \theta$

$\hat{\theta}_{m=}=\int_{0}^{\infty} \frac{\left(\Sigma_{i=1}^{n} x_{i}+\beta\right)^{(n+\alpha)} \theta^{-[(n+\alpha)+1]+1} \exp \left(-\frac{1}{\theta}\left(\sum_{i=1}^{n} x_{i}+\beta\right)\right)}{\Gamma(n+\alpha)}$

For the equation $(B .20)$, we can write $-[(n+a)+1]+1=-[(n+a-1)+1]$ And by multiplying the integral in equation (B.20) by the quantity which equals to $\mathrm{Dl}-\left(\frac{\Gamma(\mathrm{n}+\alpha-1)}{\Gamma(\mathrm{n}+\alpha-1)}\right)$, where $\Gamma($.$) is a$ gamma function.

Then, we have

$\hat{\theta}_{\mathrm{mat}}=D \cdot \int_{0}^{0} \frac{\left.\sum_{\mathrm{i}=1}^{\mathrm{n}} \mathrm{x}_{\mathrm{i}}+\beta\right)^{(\mathrm{n}+\alpha)-1+1} \theta^{-[(\mathrm{n}+\alpha-1)+1]} \exp \left(-\frac{1}{\theta}\left(\sum_{\mathrm{i}-1}^{\mathrm{n}} \mathrm{x}_{\mathrm{i}}+\beta\right)\right)}{\Gamma(\mathrm{n}+\alpha)} d \theta$

$\hat{\theta}_{\mathrm{mal}}=\frac{\Gamma(\mathrm{n}+\alpha-1)}{\Gamma(\mathrm{n}+\alpha)}\left(\sum_{i=1}^{\mathrm{n}} \mathrm{x}_{1}+\beta\right)(\mathrm{D} 2(\mathrm{x} ; \theta))$

Where $D_{2}(x, f)$ equals to

$D 2(x ; \theta)-\int_{0}^{0} \frac{\left(\sum_{i=1}^{n} x_{i}+\beta\right)^{(n+\alpha-1)} \theta^{-[(n+\alpha-1)+1]} \exp \left(-\frac{1}{\theta}\left(\sum_{i=1}^{n} x_{i}+\beta\right)\right)}{\Gamma(n+\alpha-1)} d \theta-1$. Bethe

integral of the pdf of the Invertad Gamma distribution. Then we get the Bayes estimator of $\theta$ as the following formula:

$\hat{\theta}_{\mathrm{ma}}=\frac{\Gamma(\mathrm{n}+\alpha-1)}{\Gamma(\mathrm{n}+\alpha)}\left(\sum_{i=1}^{\mathrm{n}} \mathrm{x}_{1}+\beta\right) \quad \mathrm{n}, \beta, \alpha>0$

1.5 Baves estimation using improper distribution as prior.

To obtain the Bayes' estimator under improper distribution as prior. Substituting the equation (A.26) in equation (B.3), we get:

$\hat{\theta}_{m z s}-\int_{0}^{0} \theta P_{s}(\theta \backslash x) d \theta$

$\hat{\theta}_{m z}=\int_{0}^{0} \frac{\left(\sum_{i=1}^{n} x_{i}+b\right)^{(n+a)} \theta^{-[(n+a)+1]} \exp \left(-\frac{1}{\theta}\left(\sum_{i=1}^{n} x_{i}+b\right)\right)}{\Gamma(n+a)} d \theta$ 


\section{Under Different Priors \&Two Loss Functions To Compare Bayes Estimators With Some of Classical Estimators For the Parameter of Exponential Distribution}

$\hat{\theta}_{n z=}=\int_{0}^{=} \frac{\left(\sum_{i=1}^{n} x_{i}+b\right)^{(n+a)} \theta^{-[(n+a)+1]+1} \exp \left(-\frac{1}{\theta}\left(\sum_{i=1}^{n} x_{i}+b\right)\right.}{\Gamma(n+a)} d \theta$

For the equation (B.25), we can write $-[(n+a)+1]+1=-[(n+a-1)+1]$ And by multiplying the integral in equation (B.25) by the quantity which equals to $\mathrm{El}-\left(\frac{\Gamma(\mathrm{n}+\mathrm{a}-1)}{\Gamma(\mathrm{n}+\mathrm{a}-1)}\right)$, where $\Gamma($.$) is$ a gamma function.

Then, we have

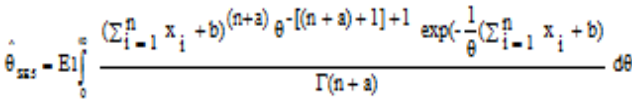

Then we have

$\hat{\theta}_{\mathrm{sas}}=\frac{\Gamma(\mathrm{n}+\mathrm{a}-1)}{\Gamma(\mathrm{n}+\mathrm{a})}\left(\sum_{\mathrm{i}-\mathrm{i}}^{\mathrm{n}} \mathrm{x}_{1}+\mathrm{b}\right)(\mathrm{B} 2(\mathrm{x} ; \theta)$

Where $E(x, \theta)$ equals to

$E x(x ; \theta)-\int_{0}^{\int} \frac{\left(\sum_{i=1}^{n} x_{i}+b\right)^{(n+2-1)} \theta^{-[(n+a-1)+1]} \exp \left(-\frac{1}{\theta}\left(\sum_{i=1}^{n} x_{i}+b\right)\right.}{\Gamma(n+a-1)} d \theta-1$. Be the

integral of the pdf of the Inverted Gamma distribution. Then we get the Bayes estimator of $\theta$ as the following formula:

$\hat{\theta}_{\mathrm{mas}}=\frac{\Gamma(\mathrm{n}+\mathrm{a}-1)}{\Gamma(\mathrm{n}+\mathrm{a})}\left(\sum_{i=1}^{\mathrm{n}} \mathrm{x}_{1}+\mathrm{b}\right) \quad \mathrm{n}, \mathrm{b}, \mathrm{a}>0$

1.6 Baves estimation using non-informative distribution as prior

To obtain the Bayes' estimator under non infortmative distribution as prior. Substituting the equation (A.32) in equation (B.3), we get:

$\hat{\theta}_{m i}=\int_{0}^{\infty} \theta P_{b}(\theta \backslash x) d \theta$

$\hat{\theta}_{m b}-\int_{0}^{e} \frac{\left(\sum_{i=1}^{n} x_{i}\right)^{(n+c-1)} \theta^{-[(n+c-1)+1]} \exp \left(-\frac{1}{\theta} \sum_{i=1}^{n} x_{i}\right)}{\Gamma(n+c-1)} d \theta$

$\hat{\theta}_{m=c}=\int_{0}^{\int} \frac{\left(\sum_{\mathrm{i}=1}^{\mathrm{n}} \mathrm{x}_{\mathrm{i}}\right)^{(\mathrm{n}+\mathrm{c}-1)} \theta^{-[(\mathrm{n}+\mathrm{c}-1)+1]+1} \exp \left(-\frac{1}{\theta} \sum_{\mathrm{i}=1}^{\mathrm{n}} \mathrm{x}_{\mathrm{i}}\right)}{\Gamma(\mathrm{n}+\mathrm{c}-1)} d \theta$

For the equation (B.30), we can write $-[(n+c-1)+1]+1=-[(n+c-2)+1]$ And by multiplying the integral in equation (B.30) by the quantity which equals to $\mathrm{F}-\left(\frac{\Gamma(\mathrm{n}+\mathrm{c}-2)}{\Gamma(\mathrm{n}+\mathrm{c}-2)}\right)$, where $\Gamma($.$) is$ a gamma function.

Then, we have

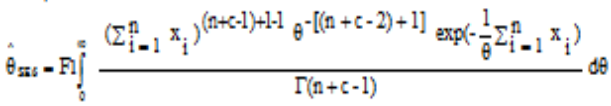

Then we have

$\hat{\theta}_{m a s}=\frac{\Gamma(n+c-2)}{\Gamma(n+c-1)}\left(\sum_{i=1}^{n} x_{i}\right)(R(x ; \theta))$
Where $F 2(x, t)$ equals to

$R(x ; \theta)-\int_{0}^{\pi} \frac{\left(\sum_{i=1}^{n} x_{i}\right)^{(n+c-2)} \theta^{-[(n+c-2)+1]} \exp \left(-\frac{1}{\theta} \sum_{i=1}^{n} x_{i}\right)}{\Gamma(n+c-2)} d \theta-1$. Be the integral of the

pdf of the Inverted Gamma distribution. Then we get the Bayes estimator of $\theta$ as the following formula:

$\hat{\theta}_{\mathrm{ma} 6}=\frac{\Gamma(\mathrm{n}+\mathrm{c}-2)}{\Gamma(\mathrm{n}+\mathrm{c}-1)}\left(\sum_{\mathrm{i}=1}^{\mathrm{n}} \mathrm{x}_{\mathrm{i}}\right) \quad \mathrm{n}, \mathrm{c}>0$

Appendix-C: The following is the derivation of these estimators under the weighted squared error loss function.

1. The weighted squared error loss function

To obtain the Bayes' estimator, we minimize the posterior expected loss given by:

$\mathrm{L}_{2}(\hat{\theta}, \theta)-\frac{(\theta-\theta)^{2}}{\theta}$, the risk function is:

$R_{2}\left(\frac{(\hat{\theta}-\theta)^{2}}{\theta}\right)-E\left[L_{2}(\theta, \theta)\right]$

$R_{2}\left(\frac{(\hat{\theta}-\theta)^{2}}{\theta}\right)-\int_{\theta} L_{2}(\theta, \theta) P(\theta \backslash x) d \theta \Rightarrow R_{2}\left(\frac{(\hat{\theta}-\theta)^{2}}{\theta}\right)-\int_{\theta} \frac{(\theta-\theta)^{2}}{\theta} P(\theta \backslash x) d \theta$

$R_{2}\left(\frac{(\hat{\theta}-\theta)^{2}}{\theta}\right)-\int \frac{\left(\theta^{2}-2 \theta \theta+\theta^{2}\right)}{\theta} \mathbb{P}(\theta<x) d \theta$

$R_{2}\left(\frac{(\hat{\theta}-\theta)^{2}}{\theta}\right)-\int\left(\frac{\theta^{2}}{\theta}-2 \hat{\theta}+\theta\right) \mathrm{P}(\theta \backslash \mathrm{x}) d \theta$

$R_{2}\left(\frac{(\theta-\theta)^{2}}{\theta}\right)=\hat{\theta}^{2} \int_{\theta}^{1} \frac{1}{\theta} P(\theta \backslash x) d \theta-2 \theta \int_{\theta} P(\theta \backslash x) d \theta+\int_{\theta} \theta P(\theta \backslash x) d \theta$

$R_{2}\left(\frac{(\theta-\theta)^{2}}{\theta}\right)-\hat{\theta}^{2} \mathrm{E}\left(\frac{1}{\theta} \backslash \mathrm{x}\right)-2 \hat{\theta}+\mathrm{E}(\theta \mid \mathrm{x})$

Lat $\frac{\partial}{\lambda} R_{2}\left(\frac{(\hat{\theta}-\theta)^{2}}{\theta}\right)=0$, we get Bayes estimator of $\theta$ denoted by $\hat{\theta}_{\mathrm{wan}}$ for the above prior $8 \theta$ as follows

$\hat{\theta}_{\operatorname{vas}}=\frac{1}{\mathrm{E}\left(\frac{1}{\theta} \backslash \mathrm{x}\right)}=\frac{1}{\int_{\theta}^{\frac{1}{\theta}} \frac{1}{\theta} \mathrm{P}(\theta \backslash \mathrm{x}) \mathrm{d \theta}}$

1.1 Baves estimation using Lerv distribution as prior

To obtain the Bayes' estimator under Lavy distribution as prior. Substituting the equation (A.6) in the integral in equation (C.3), we get:

$\mathrm{E}\left(\frac{1}{\theta} \backslash \mathrm{x}\right)-\int_{0}^{e} \frac{1}{\theta} \mathrm{P}_{i}(\theta \backslash \mathrm{x}) \mathrm{d \theta}$ 


\section{Under Different Priors \&Two Loss Functions To Compare Bayes Estimators With Some of Classical Estimators For the \\ Parameter of Exponential Distribution}

$\mathrm{E}\left(\frac{1}{\theta} \backslash \mathrm{x}\right)-\int_{0}^{\infty} \frac{1}{\theta} \frac{\left(\sum_{\mathrm{i}=1}^{\mathrm{n}} \mathrm{x}_{\mathrm{i}}+\frac{\mathrm{b}_{2}}{2}\right)^{\left(\mathrm{n}+\frac{1}{2}\right)}}{\Gamma\left(\mathrm{n}+\frac{1}{2}\right)} \theta^{\left[\left[\left(\mathrm{n}+\frac{1}{2}\right)+1\right]\right.} \exp \left(-\frac{1}{\theta}\left(\sum_{i=1}^{\mathrm{n}} \mathrm{x}_{1}+\frac{b_{i}}{2}\right)\right) d \theta$

$\mathrm{E}\left(\frac{1}{\theta} \backslash \mathrm{x}\right)-\int_{0}^{0} \frac{\left(\sum_{\mathrm{i}-1}^{\mathrm{n}} \mathrm{x}_{\mathrm{i}}+\frac{\mathrm{b}_{z}}{2}\right)^{\left(\mathrm{a}+\frac{1}{2}\right)}}{\Gamma\left(\mathrm{n}+\frac{1}{2}\right)} \theta^{\left[\left(\mathrm{n}+\frac{1}{2}\right)+1\right\}-1} \exp \left(-\frac{1}{\theta}\left(\sum_{i=1}^{\mathrm{n}} \mathrm{x}_{1}+\frac{\mathrm{b}_{2}}{2}\right)\right) d \theta$

For the equation (C.5), we can write

$-\left[\left(\mathrm{n}+\frac{1}{2}\right)+1\right]-1--\left[\mathrm{n}+\frac{1}{2}+1+1\right]-\left[\left(\mathrm{n}+\frac{3}{2}\right)+1\right]$. By multiplying the integral in equation (C.5) by the quantity which equals to $\mathrm{Al}=\left(\frac{\Gamma\left(\mathrm{n}+\frac{3}{2}\right)}{\Gamma\left(\mathrm{n}+\frac{3}{2}\right)}\right)$, where $\Gamma($.$) is a gamma function$

Then, we have

$E\left(\frac{1}{\theta} \backslash x\right)-A 1 \int_{0}^{0} \frac{\left(\sum_{i=1}^{n} x_{i}+\frac{b_{i}}{2}\right)^{\left(n+\frac{1}{2}\right)+1-1}}{\Gamma\left(n+\frac{1}{2}\right)} \theta^{\left\{\left(n+\frac{3}{2}\right)+1\right]} \exp \left(-\frac{1}{\theta}\left(\sum_{i=1}^{n} x_{1}+\frac{b_{i}}{2}\right)\right) d \theta$

then, we have

$\mathrm{E}\left(\frac{1}{\theta} \backslash \mathrm{x}\right)=\frac{\Gamma\left(\mathrm{n}+\frac{3}{2}\right)}{\Gamma\left(\mathrm{n}+\frac{1}{2}\right)}\left(\sum_{;-1}^{\mathrm{n}} \mathrm{x}_{1}+\frac{\mathrm{b}_{2}}{2}\right)(\mathrm{A} 2(\mathrm{x} ; \theta))$

Where $A(x, \theta)$ equals to

$A(x ; \theta)-\int_{0}^{\infty} \frac{\left(\Sigma_{i=1}^{n} x_{i}+\frac{b_{z}}{2}\right)^{\left(n+\frac{3}{2}\right)}}{\Gamma\left(n+\frac{3}{2}\right)} \theta^{\left[\left(n+\frac{3}{2}\right)+1\right]} \exp \left(-\frac{1}{\theta}\left(\sum_{i=1}^{n} x_{1}+\frac{b_{z}}{2}\right)\right) d \theta-1$. Be the integral of the pdf of the Inverted Gamma distribution. Then we get the Bayes estimator of $\theta$ as the following formula:

$$
\mathrm{E}\left(\frac{1}{\theta} \backslash \mathrm{x}\right)=\frac{\Gamma\left(\mathrm{n}+\frac{3}{2}\right)}{\Gamma\left(\mathrm{n}+\frac{1}{2}\right)\left(\sum_{\mathrm{i}=1}^{\mathrm{n}} \mathrm{x}_{\mathrm{i}}+\frac{\mathrm{b}_{3}}{2}\right)} \quad, \mathrm{n} \& \mathrm{~b},>0
$$

Substituting the equation (C.8) in equation (C.3), we get:

$\hat{\theta}_{w=1}=\frac{\Gamma\left(n+\frac{1}{2}\right)\left(\sum_{i=1}^{n} x_{i}+\frac{b_{z}}{2}\right)}{\Gamma\left(n+\frac{3}{2}\right)} \quad, n \& b_{j}>0$

1.2 Baves estimation using Gumbel tope-II distribution as prior.

To obtain the Bayas' estimator under the Gumbel type-I distribution as prior. Substituting the equation (A.12) in the integral in equation (C.3), we get:
$E\left(\frac{1}{\theta} \backslash x\right)-\int_{0}^{0} \frac{1}{\theta} P_{2}(\theta \backslash x) d \theta$

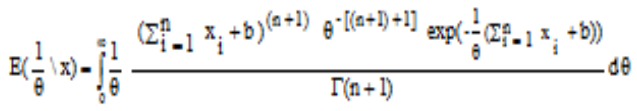

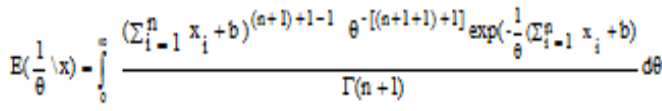

For the equation (C.11), we can write- $[(n+1+1)+1]=-[(n+2)+1]$. And by multiplying the integral in equation (C.11) by the quantity which equals to $\mathrm{B}-\left(\frac{\Gamma(\mathrm{n}+2)}{\Gamma(\mathrm{n}+2)}\right)$, where $\Gamma($.$) is a$ gamma function

Then, we have

$\mathrm{E}\left(\frac{1}{\theta} \backslash \mathrm{x}\right)-\mathrm{B}: \int_{0}^{\int} \frac{\left(\sum_{\mathrm{i}=1}^{\mathrm{n}} \mathrm{x}_{\mathrm{i}}+b\right)^{(\mathrm{n}+2)-1} \theta^{-[(a+2)+1]} \exp \left(-\frac{1}{\theta}\left(\sum_{\mathrm{i}-1}^{\mathrm{p}} \mathrm{x}_{\mathrm{i}}+b\right)\right.}{\Gamma(\mathrm{n}+1)} d \theta$

Then we have

$\mathrm{E}\left(\frac{1}{\theta} \backslash \mathrm{x}\right)=\frac{\Gamma(\mathrm{n}+2)}{\Gamma(\mathrm{n}+1)\left(\sum_{\mathrm{i}=1}^{\mathrm{n}} \mathrm{x}_{\mathrm{i}}+b\right)}(\mathrm{B} 2(\mathrm{x} ; \theta))$

Where $\bar{B}_{2}(\mathrm{x}, \mathrm{\theta})$ equals to

$B 2(x ; \theta)-\int_{0}^{\int} \frac{\left(\sum_{i=1}^{n} x_{i}+b\right)^{(n+2)} \theta^{-[(n+2)+1]} \exp \left(-\frac{1}{\theta}\left(\sum_{i=1}^{A} x_{i}+b\right)\right.}{\Gamma(n+2)} d \theta-1$. Be the integral of the pdf of the Inverted Gamma distribution. Then we get the Bayes estimator of $\theta$ as the following formula:

$E\left(\frac{1}{\theta} \backslash x\right)=\frac{\Gamma(n+2)}{\Gamma(n+1)\left(\sum_{i=1}^{n} x_{i}+b\right)}, n \& b>0$

Substituting the equation (C.14) in equation (C.3), we get:

$\hat{\theta}_{\operatorname{vin}}=\frac{\Gamma(n+1)\left(\sum_{b-1}^{n} x_{i}+b\right)}{\Gamma(n+2)} \quad, n \& b>0$

1.3 Baves estimation using Inverse chi-squared distribution as prior.

To obtain the Bayes ' estimator under inverse chi-squared distribution as prior. Substituting the equation (A.16) in the integral in equation (C.3), we get:

$E\left(\frac{1}{\theta} \backslash x\right)-\int_{0}^{\infty} \frac{1}{\theta} P_{i}(\theta \backslash x) d \theta$

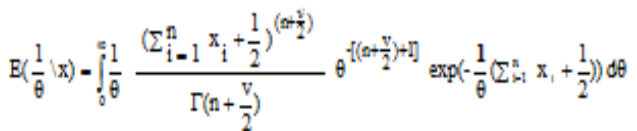

$\mathrm{E}\left(\frac{1}{\theta} \backslash \mathrm{x}\right)-\int_{0}^{=} \frac{\left(\sum_{\mathrm{i}=1}^{\mathrm{n}} \mathrm{x}_{\mathrm{i}}+\frac{1}{2}\right)^{\left(n+\frac{1}{2}\right)}}{\Gamma\left(\mathrm{n}+\frac{\mathrm{y}}{2}\right)} \theta^{\left[\left(n+\frac{y}{2}\right)+\mathrm{n}\right]-1} \exp \left(-\frac{1}{\theta}\left(\sum_{i=1}^{\mathrm{n}} \mathrm{x}_{1}+\frac{1}{2}\right)\right) d \theta$ 


\section{Under Different Priors \&Two Loss Functions To Compare Bayes Estimators With Some of Classical Estimators For the \\ Parameter of Exponential Distribution}

For the equation (C.17), we can write $-\left[\left(\left(\mathrm{n}+\frac{v}{2}\right)+1\right)-1-\left[\left(\left(\mathrm{n}+\frac{v}{2}+1\right)+1\right]\right.\right.$ And by multiplying the integral in equation (C.17) by the quantity which equals to $\mathrm{Cl}=\left(\frac{\Gamma\left(\mathrm{n}+\frac{v}{2}+1\right)}{\Gamma\left(\mathrm{n}+\frac{v}{2}+1\right)}\right)$, where $\Gamma($.$) is a$ gemma function.

Then, we have

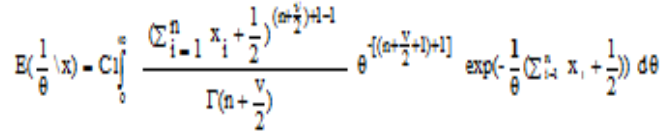

Then we have

$\mathrm{E}\left(\frac{1}{\theta} \backslash \mathrm{x}\right)=\frac{\Gamma\left(\mathrm{n}+\frac{\mathrm{v}}{2}+1\right)}{\Gamma\left(\mathrm{n}+\frac{\mathrm{v}}{2}\right)\left(\sum_{\mathrm{i}=1}^{\mathrm{n}} \mathrm{x}_{\mathrm{i}}+\frac{1}{2}\right)}(\mathrm{C} 2(\mathrm{x} ; \theta))$

Where $\mathrm{C} 2(\mathrm{x}, \mathrm{t})$ equals to

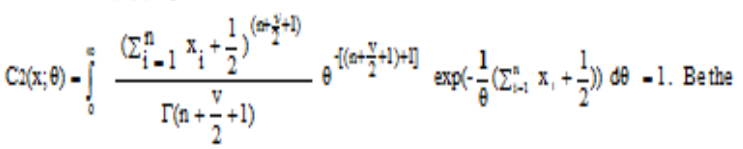

integral of the pdf of the Inverted Gemma distribution. Then we gat the Bayes estimator of $\theta$ as the following formula:

$\mathrm{E}\left(\frac{1}{\theta} \backslash \mathrm{x}\right)=\frac{\Gamma\left(\mathrm{n}+\frac{\mathrm{v}}{2}+1\right)}{\Gamma\left(\mathrm{n}+\frac{\mathrm{v}}{2}\right)\left(\sum_{\mathrm{i}=1}^{\mathrm{n}} \mathrm{x}_{\mathrm{i}}+\frac{1}{2}\right)} \quad \mathrm{n}, \mathrm{v}>0$

Substituting the equation (C.20) in equation (C.3), we get:

$\hat{\theta}_{\operatorname{vin}}=\frac{\Gamma\left(\mathrm{n}+\frac{V}{2}\right)\left(\sum_{i=1}^{\mathrm{n}} \mathrm{x}_{\mathrm{i}}+\frac{1}{2}\right)}{\Gamma\left(\mathrm{n}+\frac{\mathrm{v}}{2}+1\right)} \quad \mathrm{n}, \mathrm{v}>0$

1.4 Baves estimation using Inverted gamma distribution as prior.

To obtain the Bayes' estimator under the invertad gamma distribution as prior. Substituting the equation (A.21) in the integral in equation (C.3), we get:

$E\left(\frac{1}{\theta} \backslash x\right)-\int_{0}^{\infty} \frac{1}{\theta} P_{s}(\theta \backslash x) d \theta$

$E\left(\frac{1}{\theta} \mid x\right)-\int_{i}^{\infty} \frac{\left(\Sigma_{i=1}^{n} x_{i}+\beta\right)^{(n+\alpha)} \theta^{-[(n+\alpha)+1]} \exp \left(-\frac{1}{\theta}\left(\Sigma_{i=1}^{n} x_{i}+\beta\right)\right)}{\Gamma(n+\alpha)} d \theta$

$E\left(\frac{1}{\theta} \mid x\right)=\int_{0}^{e} \frac{\left(\sum_{i=1}^{n} x_{i}+\beta\right)^{(n+\alpha)} \theta^{-[(n+\alpha)+1]-1} \exp \left(-\frac{1}{\theta}\left(\sum_{i=1}^{n} x_{i}+\beta\right)\right)}{\Gamma(n+\alpha)}$
For the equation (C.23), we can write $-[(n+a)+1]-1=-[(n+a+1)+1]$ And by multiplying the integral in equation (C.23) by the quantity which equals $D 1-\left(\frac{\Gamma(n+\alpha+1)}{\Gamma(n+\alpha+1)}\right)$, where $\Gamma($.$) is a$ gamma function.

Then, we have

$E\left(\frac{1}{\theta} \backslash x\right)-D i \int_{0}^{=} \frac{\left(\sum_{i=1}^{\mathrm{n}} x_{i}+\beta\right)^{(n+\alpha)-1+1} \theta^{-[(n+\alpha+1)+1]} \exp \left(-\frac{1}{\theta}\left(\sum_{i=1}^{\mathrm{n}} \mathrm{x}_{\mathrm{i}}+\beta\right)\right)}{\Gamma(\mathrm{n}+\alpha)} d \theta$.

Then we have

$\mathrm{E}\left(\frac{1}{\theta} \backslash \mathrm{x}\right)=\frac{\Gamma(\mathrm{n}+\alpha+1)}{\Gamma(\mathrm{n}+\alpha)\left(\sum_{\mathrm{i}-1}^{\mathrm{n}} \mathrm{x}_{\mathrm{i}}+\beta\right)}(\mathrm{D} 2(\mathrm{x} ; \theta))$

Where $D_{2}(x, \theta)$ equals to

$D 2(x ; \theta)-\int_{0}^{0} \frac{\left(\sum_{i=1}^{n} x_{i}+\beta\right)^{(n+\alpha+1)} \theta^{-[(n+\alpha+1)+1]} \exp \left(-\frac{1}{\theta}\left(\sum_{i=1}^{n} x_{i}+\beta\right)\right)}{\Gamma(n+\alpha+1)} d \theta-1$. Bethe

integral of the pdf of the Inverted Gamma distribution. Then we gat the Bayes estimator of $\theta$ as the following formula:

$\mathrm{E}\left(\frac{1}{\theta} \backslash \mathrm{x}\right)=\frac{\Gamma(\mathrm{n}+\alpha+1)}{\Gamma(\mathrm{n}+\alpha)\left(\sum_{\mathrm{i}-1}^{\mathrm{n}} \mathrm{x}_{\mathrm{i}}+\beta\right)} \quad \mathrm{n}, \beta, \alpha>0$

Substituting the equation (C.26) in equation (C.3), we get:

$\hat{\theta}_{v \mathbf{m a} \alpha}=\frac{\Gamma(\mathrm{n}+\alpha)\left(\sum_{i=1}^{n} \mathrm{x}_{1}+\beta\right)}{\Gamma(\mathrm{n}+\alpha+1)} \quad \mathrm{n}, \beta, \alpha>0$

1.5 Baves estimation using improper distribution as prior.

To obtain the Baysa' estimator under improper distribution as prior. Substituting the equation (A.26) in the integral in equation (C.3), we get:

$\mathrm{E}\left(\frac{1}{\theta} \backslash \mathrm{x}\right)-\int_{0}^{\pi} \frac{1}{\theta} \mathrm{P}_{s}(\theta \backslash \mathrm{x}) d \theta$

$E\left(\frac{1}{\theta} \backslash x\right)-\int_{0}^{e} \frac{1}{\theta} \frac{\left(\sum_{i=1}^{n} x_{i}+b\right)^{(n+a)} \theta^{-[(n+a)+1]} \exp \left(-\frac{1}{\theta}\left(\sum_{i=1}^{n} x_{i}+b\right)\right)}{\Gamma(n+a)} d \theta$

$E\left(\frac{1}{\theta} \backslash x\right)-\int_{0}^{0} \frac{\left(\sum_{i=1}^{n} x_{i}+b\right)^{(n+a)} \theta^{-[(n+a)+1]-1} \exp \left(-\frac{1}{\theta}\left(\sum_{i=1}^{n} x_{i}+b\right)\right)}{\Gamma(n+a)} d \theta$

For the equation (C.29), we can write $-[(n+a)+1]-1=-[(n+a+1)+1]$ And by multiplying the integral in equation (C.29) by the quantity which equals to $\mathrm{El}-\left(\frac{\Gamma(\mathrm{n}+\mathrm{a}+1)}{\Gamma(\mathrm{n}+\mathrm{a}+1)}\right)$, where $\Gamma($.$) is a$ gamma function.

Then, we have

$E\left(\frac{1}{\theta} \backslash x\right)-E \cdot \int_{0}^{0} \frac{\left(\sum_{i=1}^{n} x_{i}+b\right)^{(n+3)+1-1} \theta^{-[(n+a+1)+1]} \exp \left(-\frac{1}{\theta}\left(\sum_{i=1}^{n} x_{i}+b\right)\right)}{\Gamma(n+a)} d \theta$ 


\section{Under Different Priors \&Two Loss Functions To Compare Bayes}

Estimators With Some of Classical Estimators For the

Parameter of Exponential Distribution

$E\left(\frac{1}{\theta} \backslash x\right)-\frac{\Gamma(n+a+1)}{\Gamma(n+a)\left(\Sigma_{i=1}^{n} x_{i}+b\right)}(E 2(x ; \theta))$

Where $E_{2}(x, \theta)$ equals to

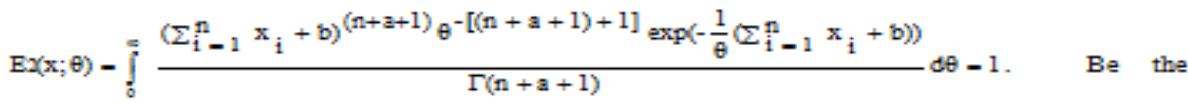

integral of the pdf of the Inverted Gamma distribution. Then we get the Bayes estimator of $\theta$ as the following formula:

$\mathrm{E}\left(\frac{1}{\theta} \backslash \mathrm{x}\right)-\frac{\Gamma(\mathrm{n}+\mathrm{a}+1)}{\Gamma(\mathrm{n}+\mathrm{a})\left(\Sigma_{\mathrm{i}-1}^{\mathrm{n}} \mathrm{x}_{\mathrm{i}}+\mathrm{b}\right)} \quad \mathrm{n}, \mathrm{b}, \mathrm{a}>0$

Subatituting the equation (C.32) in equation (C.3), we get:

$\hat{\theta}_{\text {wass }}-\frac{\Gamma(\mathrm{n}+\mathrm{a})\left(\sum_{t-1}^{\mathrm{n}} \mathrm{x}_{1}+\mathrm{b}\right)}{\Gamma(\mathrm{n}+\mathrm{a}+1)}$

$\mathrm{n}, \mathrm{b}, \mathrm{a}>0$

1.6 Baves estimation using non-informative distribution as prior

To obtain the Bayes' estimator under non infortmative distribution as prior. Substituting the equation (A.32) in the integral in equation (C.3), we get:

$\mathrm{E}\left(\frac{1}{\theta} \backslash \mathrm{x}\right)-\int_{0}^{\pi} \frac{1}{\theta} \mathrm{P}_{6}(\theta>\mathrm{x}) \mathrm{d \theta}$

$E\left(\frac{1}{\theta} \backslash \mathrm{x}\right)-\int_{0}^{\infty} \frac{1}{\theta} \frac{\left(\sum_{i-1}^{n} x_{i}\right)^{(n+c-1)} \theta^{-[(n+c-1)+1]} \exp \left(-\frac{1}{\theta} \sum_{i-1}^{n} x_{i}\right)}{\Gamma(n+c-1)} d \theta$

$E\left(\frac{1}{\theta} \backslash \mathrm{x}\right)-\int_{0}^{\infty} \frac{\left(\sum_{\mathrm{i}-1}^{\mathrm{n}} \mathrm{x}_{\mathrm{i}}\right)^{(\mathrm{n}+\mathrm{c}-1)} \theta^{-[(\mathrm{n}+\mathrm{c}-1)+1]-1} \exp \left(-\frac{1}{\theta} \sum_{\mathrm{i}-1}^{\mathrm{n}} \mathrm{x}_{\mathrm{i}}\right)}{\Gamma(\mathrm{n}+\mathrm{c}-1)} d \theta$

For the equation (C.35), we can write $-[(n+c-1)+1]-1=-[(n+c)+1]$ And by multiplying the integral in equation (C.35) by the quantity which equals to $F-\left(\frac{\Gamma(n+c)}{\Gamma(n+c)}\right)$, where $\Gamma($.$) is a$ gamma function.

Then, we have

$\mathrm{E}\left(\frac{1}{\theta} \backslash \mathrm{x}\right)-\mathrm{F} \int_{0}^{\infty} \frac{\left(\sum_{i-1}^{n} \mathrm{x}_{i}\right)^{(n+c-1)+1-1} \theta^{-[(n+c-2)+1]} \exp \left(-\frac{1}{\theta} \sum_{i-1}^{n} x_{i}\right)}{\Gamma(n+c-1)} d \theta$

$\mathrm{E}\left(\frac{1}{\theta} ; \mathrm{x}\right)-\frac{\Gamma(\mathrm{n}+\mathrm{c})}{\Gamma(\mathrm{n}+\mathrm{c}-1)\left(\sum_{\mathrm{i}=1}^{\mathrm{n}} \mathrm{x}_{\mathrm{i}}\right)}(\mathrm{F} 2(\mathrm{x} ; \theta))$

Where $F_{2}(x, \theta)$ equals to

$F_{2}(x ; \theta)-\int_{0}^{0} \frac{\left(\sum_{i-1}^{n} x_{i}\right)^{(n+c)} \theta^{-[(n+c)+1]} \exp \left(-\frac{1}{\theta} \sum_{i-1}^{n} x_{i}\right)}{\Gamma(n+c)} d \theta-1$. Bethe integral of the pdf of the Inverted Gamma distribution. Then we get the Bayes estimator of $\theta$ as the following formula:

$E\left(\frac{1}{\theta} \backslash x\right)-\frac{\Gamma(n+c)}{\Gamma(n+c-1)\left(\sum_{i=1}^{n} x_{i}\right)}$

Substituting the equation (C.38) in equation (C.3), we get:

$\hat{\theta}_{\text {was }}-\frac{\Gamma(\mathrm{n}+\mathrm{c}-1)\left(\sum_{t-1}^{\mathrm{n}} \mathrm{x}_{1}\right)}{\Gamma(\mathrm{n}+\mathrm{c})}$

$\mathrm{n}, \mathrm{c}>\mathrm{0}$ 


\section{Under Different Priors \&Two Loss Functions To Compare Bayes Estimators With Some of Classical Estimators For the Parameter of Exponential Distribution}

Appendix-D: The following is the programs algorithm. scale parameter

Algorithm (1): To compute MLE for scale $\operatorname{parameter}(\hat{\theta})$ with MSE and MWSE.

\section{Start}

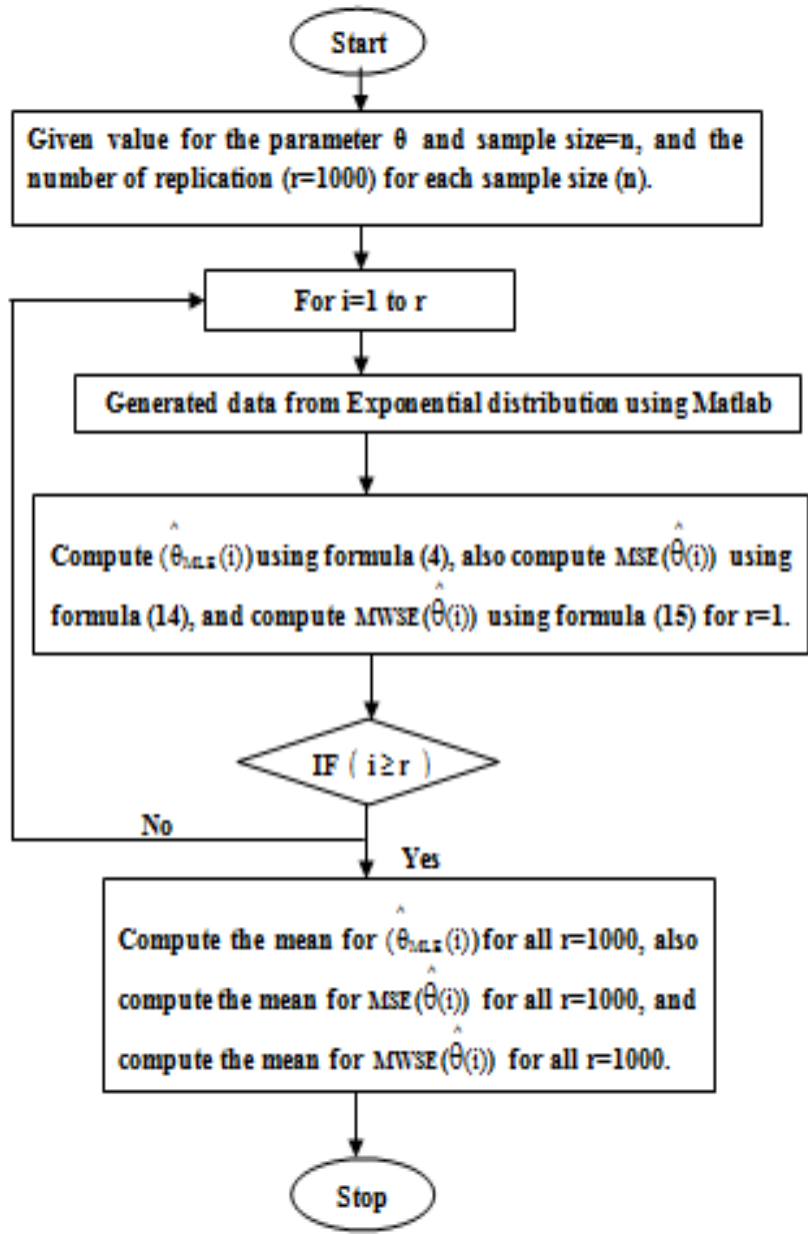

Algorithm (2): To compute MM for

$(\theta)$ with MSE and MWSE.

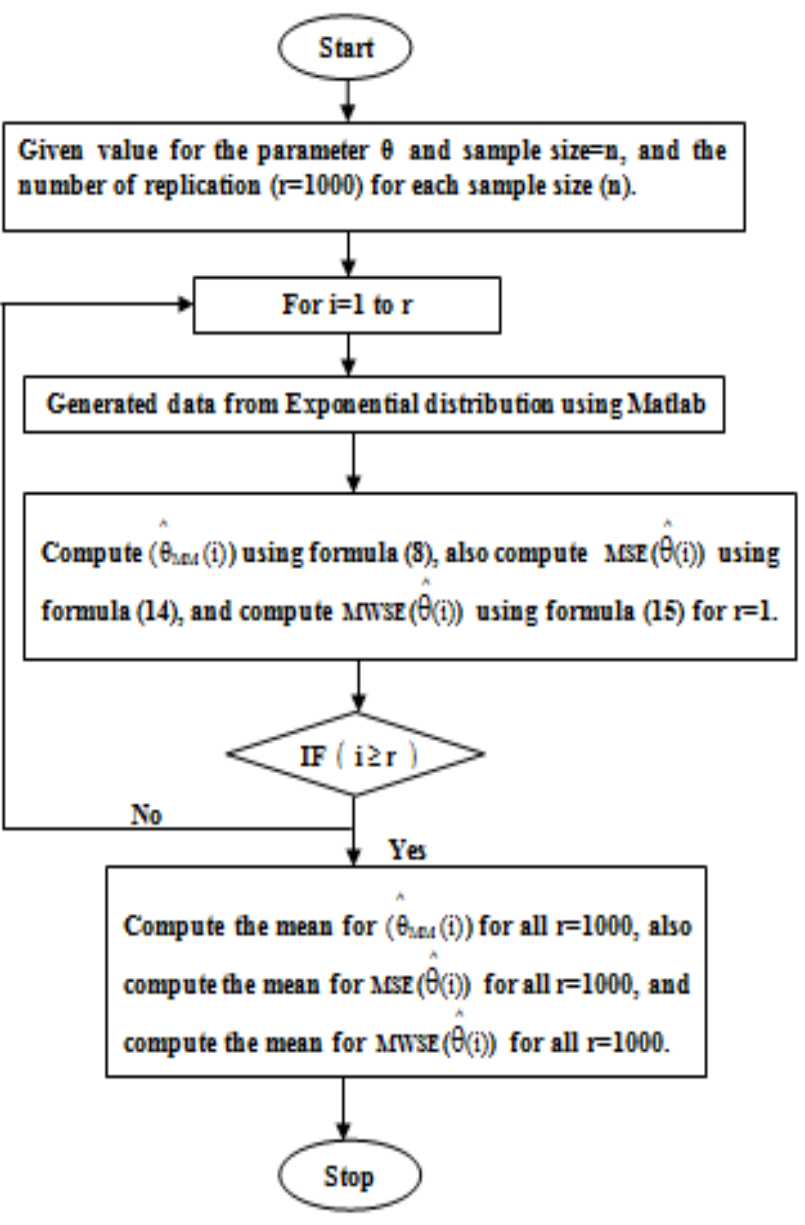




\title{
Under Different Priors \&Two Loss Functions To Compare Bayes Estimators With Some of Classical Estimators For the Parameter of Exponential Distribution
}

\begin{abstract}
Algorithm (3): To compute Bayes estimators $\left(\hat{\theta}_{\mathrm{SE} 1}\right)$ using
Algorithm (4): To compute Bayes estimators

$\left(\hat{\theta}_{\mathrm{WSE} 1}\right)$ using

Levy distribution as prior distribution for $\theta$ with MSE.

Levy distribution as prior distribution for $\theta$ with
\end{abstract}

MWSE.

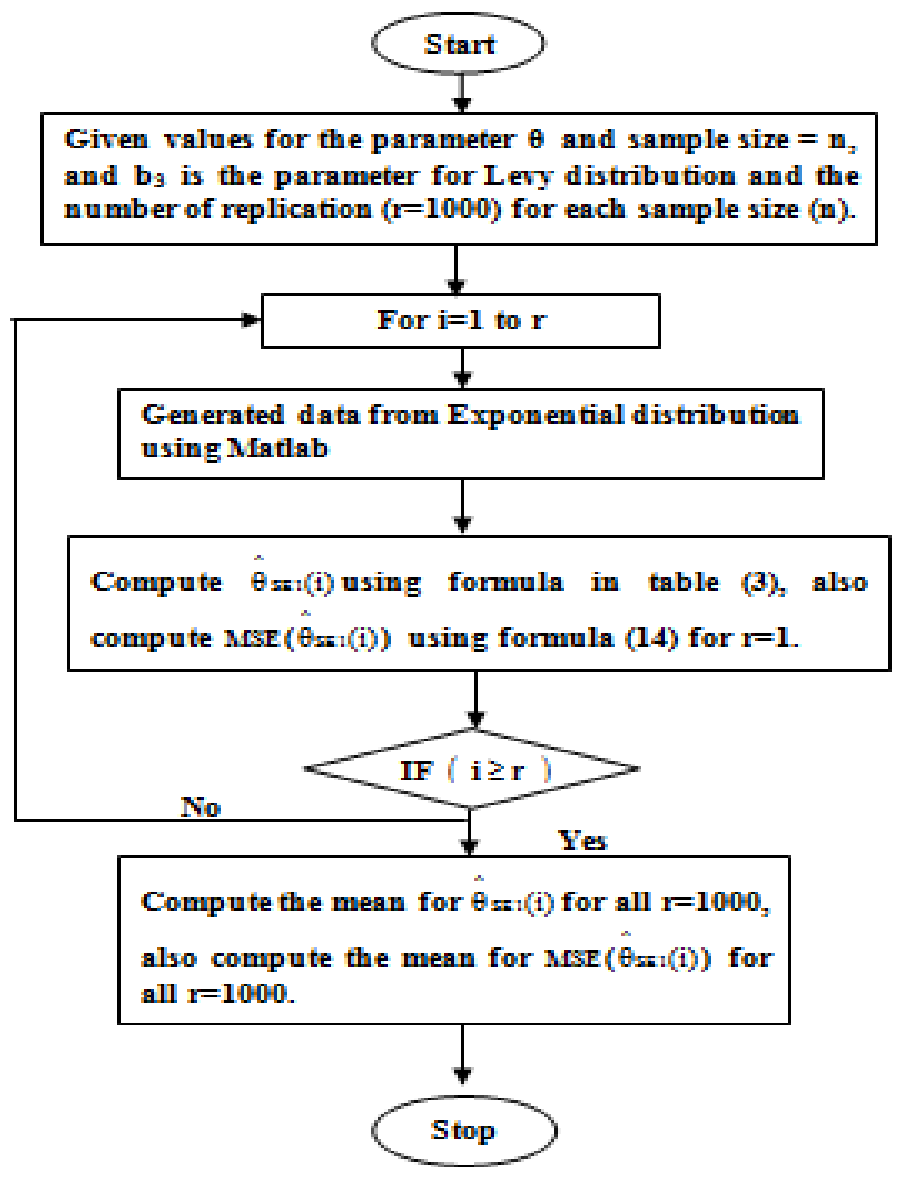

Note (1): we can reformulate the Algorithm (3) to compute Algorithm (4) to

Bayes estimators $\hat{\theta}_{\text {SEk }}, \mathrm{k}=2,3,4,5,6$ under using other

$\theta_{\text {WSEk }}, \mathrm{k}=2,3,4,5,6$ under using

distributions as prior distribution for $\theta$ with MSE

for $\theta$ with MWSE.

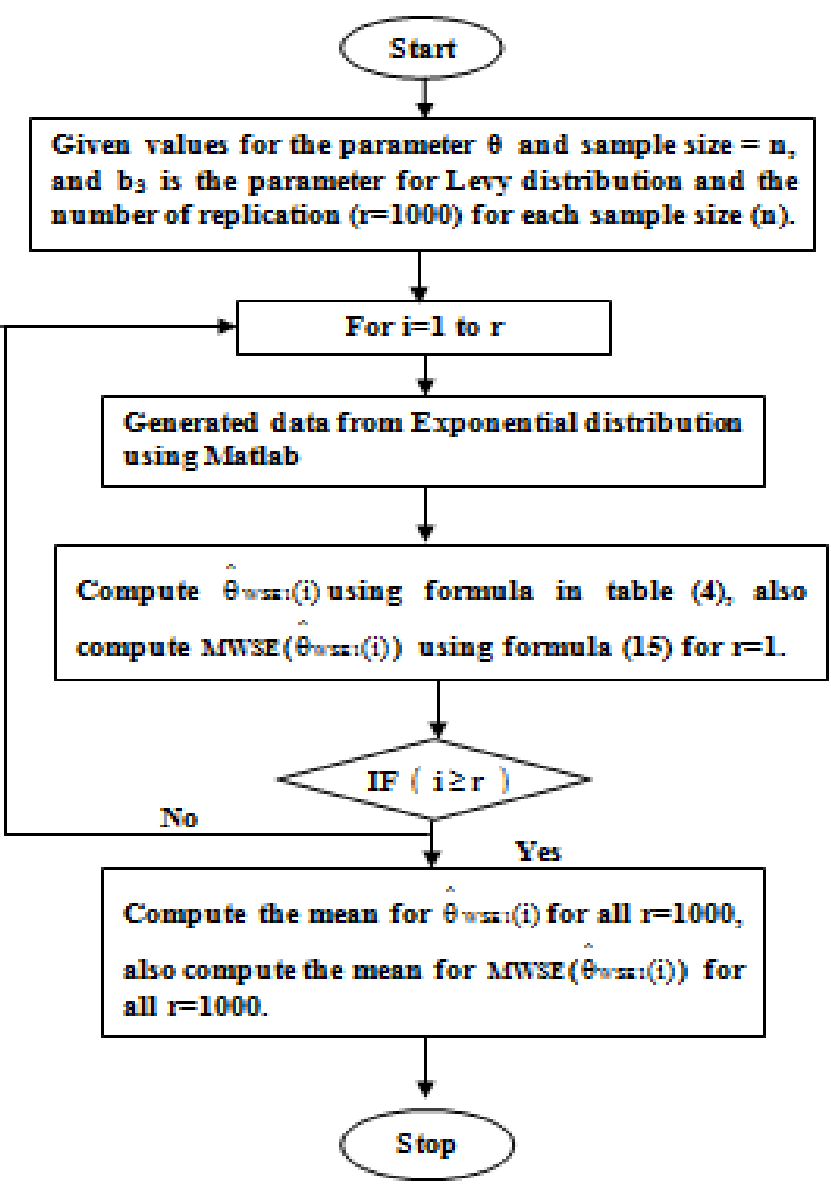

Note (2): We can reformulate the

compute Bayes estimators

other distributions as prior distribution

30 العدد (99) المجلم العلوم الاقتصاديتتوالإداريتت 2017 لسنت 2017




\section{استعمال دوال أوليت ودالتين خسارة مختلفت لمقارنتمقدروات بيز مع بعض

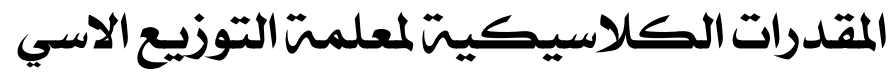

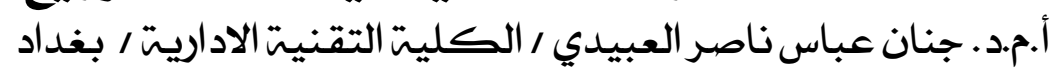

المستخلص:

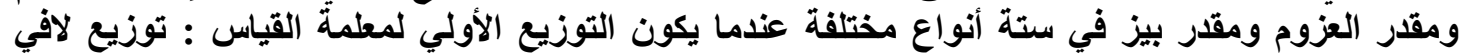

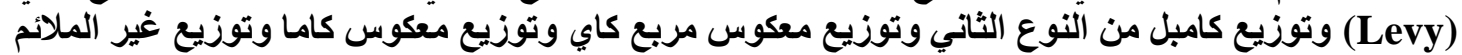
(Improper)

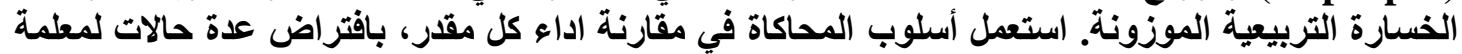

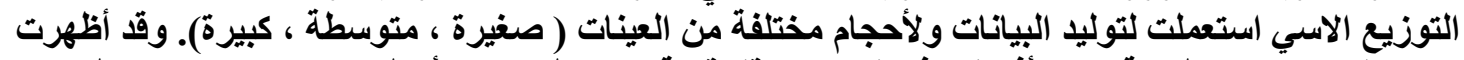

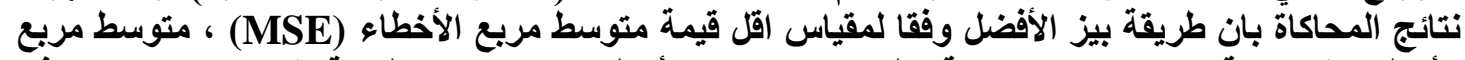

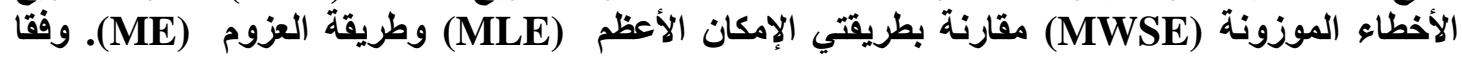
للتتائج المستحصلة ، نرى بانه عندما يكون التوزيع الاوني الاولي لـ

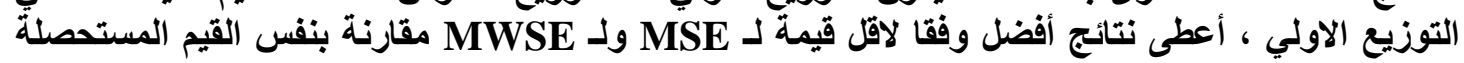

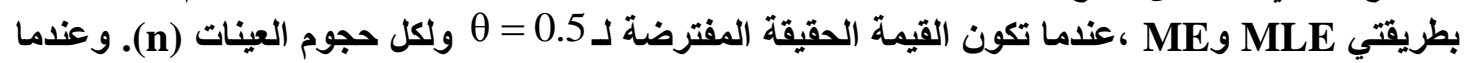

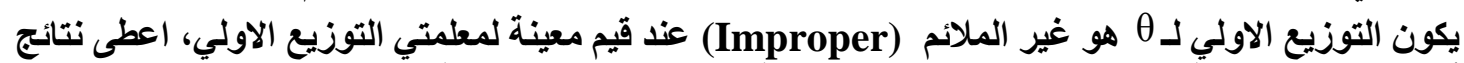

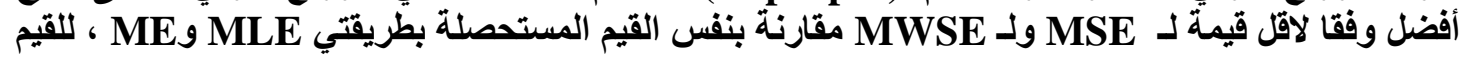

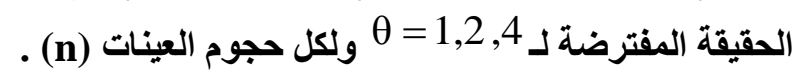

المسطحات الرئيسة للبحث/ التوزيع الآسي ، طريقة الإمكان الأعظم، طريقة العزوم ، طريقة بيز ،

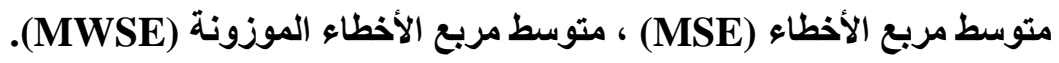

